This item was submitted to Loughborough's Research Repository by the author.

Items in Figshare are protected by copyright, with all rights reserved, unless otherwise indicated.

\title{
BRDF representation and acquisition
}

PLEASE CITE THE PUBLISHED VERSION

http://dx.doi.org/10.1111/cgf.12867

\section{PUBLISHER}

(c) The Author(s) Computer Graphics Forum (c) The Eurographics Association and John Wiley \& Sons Ltd.

Published by John Wiley \& Sons Ltd.

\section{VERSION}

AM (Accepted Manuscript)

\section{PUBLISHER STATEMENT}

This work is made available according to the conditions of the Creative Commons Attribution-NonCommercialNoDerivatives 4.0 International (CC BY-NC-ND 4.0) licence. Full details of this licence are available at: https://creativecommons.org/licenses/by-nc-nd/4.0/

\section{LICENCE}

CC BY-NC-ND 4.0

\section{REPOSITORY RECORD}

Guarnera, Dar'ya, Giuseppe C. Guarnera, Abhijeet Ghosh, Cornelia Denk, and Mashhuda Glencross. 2019. "BRDF Representation and Acquisition". figshare. https://hdl.handle.net/2134/21522. 


\title{
BRDF Representation and Acquisition
}

\author{
D. Guarnera ${ }^{1}$, G. C. Guarnera ${ }^{1}$, A. Ghosh ${ }^{2}$, C. Denk ${ }^{3}$, and M. Glencross ${ }^{1}$ \\ ${ }^{1}$ Department of Computer Science, Loughborough University \\ ${ }^{2}$ Department of Computing, Imperial College London \\ ${ }^{3}$ BMW Research, Munich
}

\begin{abstract}
Photorealistic rendering of real world environments is important in a range of different areas; including Visual Special effects, Interior/Exterior Modelling, Architectural Modelling, Cultural Heritage, Computer Games and Automotive Design.

Currently, rendering systems are able to produce photorealistic simulations of the appearance of many real-world materials. In the real world, viewer perception of objects depends on the lighting and object/material/surface characteristics, the way a surface interacts with the light and on how the light is reflected, scattered, absorbed by the surface and the impact these characteristics have on material appearance. In order to re-produce this, it is necessary to understand how materials interact with light. Thus the representation and acquisition of material models has become such an active research area.
\end{abstract}

This survey of the state-of-the-art of BRDF Representation and Acquisition presents an overview of BRDF (Bidirectional Reflectance Distribution Function) models used to represent surface/material reflection characteristics, and describes current acquisition methods for the capture and rendering of photorealistic materials.

Categories and Subject Descriptors (according to ACM CCS): I.3.3 [Computer Graphics]: Picture/Image Generation—Line and curve generation I.3.7 [Computer Graphics]: Three-Dimensional Graphics and Realism-Colour, shading, shadowing, and texture I.6.8 [Computer Graphics]: Types of simulation-Monte Carlo

\section{Introduction}

Human perception of materials depends on light that is reflected, transmitted, and/or absorbed from objects reaching the viewer [DR05]. The appearance of materials may vary significantly depending on a wide range of properties such as colour, smoothness, geometry, roughness, reflectance and angle from which the material is viewed and lighting directions. A major challenge in computer graphics is how to simply and accurately measure the appearance of material characteristics from real-world objects and implement practical editable synthetic materials accurately matching the appearance of the original.

Currently, no up to date universal material model that can represent leather, fabric, car paint, wood, plastic, rubber, mirrored surfaces, switchable surfaces etc. exists. [SDSG13]).

A variety of rendering systems are used in the software pipeline resulting in a need for optimised material representations. This demands both a flexible acquisition process and representation methods. In terms of representation and acquisition of material models, the following challenges still persist:

- No widely adopted solution
- Few solutions acquire material models that are good enough for a wide range of commercial applications without significant labour and money

- No standardised material model formats from acquisition setups

- Little standardisation across renderers, with different renderers supporting subsets of material properties

- Material models are hard to edit by artists

- Acquired material models have a high memory footprint which limits applicability

The above issues justify the need for further research in the area. In this state of the art report, we focus on current Bidirectional Reflectance Distribution Functions (BRDF) representations [NRH*77]. We describe: BRDF Models, Acquisition Methods and Limitations.

\section{Definition of the BRDF and related reflectance functions}

The BRDF is a radiometric function, currently used to varying levels of accuracy in photorealistic rendering systems. It describes, in the general case, how incident energy is redirected in all directions across a hemisphere above the surface. However, the uptake of ac- 


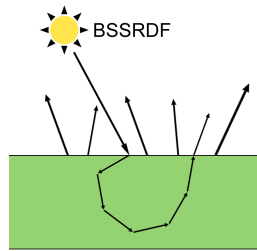

(a) BSSRDF

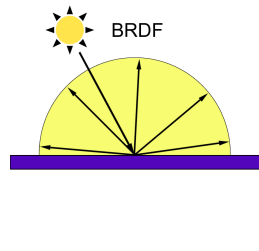

(b) BRDF

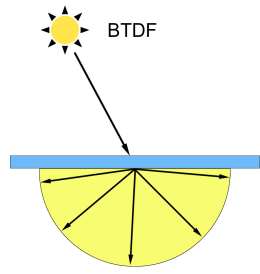

(c) BTDF
Figure 1: Scattering of light in (a) BSSRDF, (b) BRDF and (c) $B T D F$.

quired models has not been widespread across rendering packages due to their data and storage requirements. Researchers have measured hundreds of BRDFs (isotropic and anisotropic), suggested implementation techniques and allowed user input to edit and enhance materials. Recent implementations have expanded material libraries, but have not improved significantly upon material representation efficiency. Results are often obtained at the expense of time-consuming effort on a per-material basis.

Historically, the BRDF was defined and suggested over the more generalised BSSRDF (Bidirectional Scattering- Surface Reflectance-Distribution function) [JMLH01] by Nicodemus $\left[\mathrm{NRH}^{*} 77\right]$, as a simplified reflectance representation for opaque surfaces: the BRDF assumes that light entering a material leaves the material at the same position, whereas the BSSRDF can describe light transport between any two incident rays on a surface (see Figure 1(a) and 1(b)). Many common translucent materials like milk, skin and alabaster cannot be represented by a BRDF since they are characterised by their subsurface scattering behavior that smooth the surface details, with the light shining through them [GLL*04]. These materials are expensive to render and many techniques have been proposed [JMLH01,DS03,HBV03, DWd ${ }^{*} 08$, DI11, KRP*15].

The BRDF is defined as the ratio of incoming irradiance to outgoing radiance:

$$
f_{r}\left(\mathbf{v}_{\mathbf{i}}, \mathbf{v}_{\mathbf{r}}\right)=\frac{d L_{r}\left(\mathbf{v}_{\mathbf{r}}\right)}{d E_{i}\left(\mathbf{v}_{\mathbf{i}}\right)}=\frac{d L_{r}\left(\mathbf{v}_{\mathbf{r}}\right)}{L_{i}\left(\mathbf{v}_{\mathbf{i}}\right) \cos \theta_{i} d \omega_{i}}
$$

where $\mathbf{v}_{\mathbf{i}}$ and $\mathbf{v}_{\mathbf{r}}$ are vectors describing the incident $(i)$ and exitant $(r)$ directions, $E_{i}$ is incident irradiance (i.e. the incident flux per unit area of the surface), $L_{i}$ is incident radiance (i.e. the reflected flux per unit area per unit solid angle) and $L_{r}$ is the reflected radiance; the units of a BRDF are inverse steradian $[1 / s r]$. Figure 2 shows the geometry of the BRDF and the vectors used for parameterisation:

- $\mathbf{n}$ is the normal at a specific point $p$ on the surface

- $\mathbf{t}$ is the tangent vector. It is perpendicular to the normal $\mathbf{n}$ and hence it is tangent to the surface at $p$.

- $\mathbf{b}$ is the bi-tangent vector, defined as $\mathbf{b}=\mathbf{n} \times \mathbf{t}$. In literature it is also named as binormal vector.

- $\mathbf{h}$ is the halfway vector [Rus98], defined as: $\mathbf{h}=\frac{\left(\mathbf{v}_{\mathbf{i}}+\mathbf{v}_{\mathbf{r}}\right)}{\left\|\mathbf{v}_{\mathbf{i}}+\mathbf{v}_{\mathbf{r}}\right\|}$

The vectors $\mathbf{t}, \mathbf{b}$ and $\mathbf{n}$ define a local reference frame; another possibility is to have a local reference frame in which one of the axis is aligned with $\mathbf{h}$ and the other two are given by $\mathbf{b}^{\prime}=\frac{(\mathbf{n} \times \mathbf{h})}{\|\mathbf{n} \times \mathbf{h}\|}$

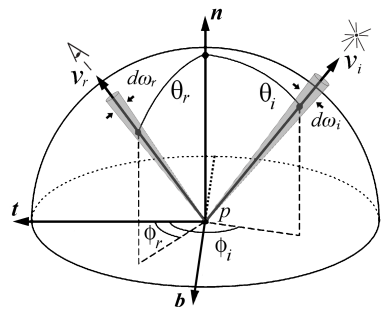

(a) BRDF as a function of $\left(\mathbf{v}_{\mathbf{i}}, \mathbf{v}_{\mathbf{r}}\right)$

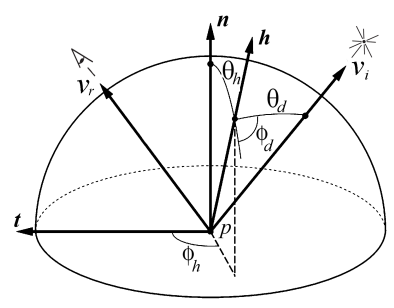

(b) Halfway vector $(h)$ and difference angle $\left(\theta_{d}, \phi_{d}\right)$
Figure 2: Geometry of surface reflection and BRDF parameterisation. Figure adapted by the authors from [Rus98].

and $\mathbf{t}^{\prime}=\mathbf{b}^{\prime} \times \mathbf{h}$. There exist other coordinate system and parameterisation, especially suited for dimensionality reduction of some isotropic BRDF models, for instance the barycentric coordinate system with respect to a triangular support proposed by Stark et al. [SAS05], or the hybrid model described by Barla et al. which could lead to a better repartition of samples to cover most of the effects of materials [BBP15].

The BRDF aims to represent the reflectance characteristics of homogeneous materials. An extension of the BRDF concept to nonhomogeneous materials is given by the Spatially Varying BRDF (SVBRDF), which can be viewed as a spatial collection of BRDFs distributed over the surface, to simulate the appearance of smooth materials [HF13]. The SVBRDF parameterisation includes extra parameters with respect to the BRDF, to take into account the location over the surface: $f_{r}\left(x, y, \mathbf{v}_{\mathbf{i}}, \mathbf{v}_{\mathbf{r}}\right)$. Capturing a SVBRDF generally requires long measurements and processing and can require large, specialised and sometimes expensive hardware rigs to capture the reflectance data. SVBRDF models represent surfaces that are nearly flat and opaque, since the model is restricted by the BRDF reciprocity and energy conservation properties and can not capture subsurface scattering.

Many real world surfaces exhibit local variations not only in reflectance but also in small-scale geometry, causing mesoscopic effects like inter-reflections, self-occlusions and self-masking, not captured by a SVBRDF representation [HF13]. Dana et $a l$. [DVGNK99] suggested that the BRDF is suitable to characterise surface variation at a coarse scale and introduced the term BTF (Bidirectional Texture Function), an image-based representation that includes small-scale geometry and can describe a fine-scale appearance of a rough surface. The aforementioned mesoscopic effects are difficult to quantify and separate from the measured data, hence BTF acquisition generally needs a large number of samples of the surface as well as high-end hardware support, due to lengthy acquisition times and storage demands [HF13]. Nevertheless, there exist low cost acquisition setups, like the kaleidoscopic device by Han and Perlin [HP03] (see Section 4.6) or the more recent mechanical gantry with rotating arms by Filip et al. [FVK14], built using a toy construction set. BTFs generally result in very realistic material appearance, since BTFs capture spatial variations of the whole surface, unlike BRDFs. Despite of this, many BTF datasets can be approximated as a sparse linear combination of 


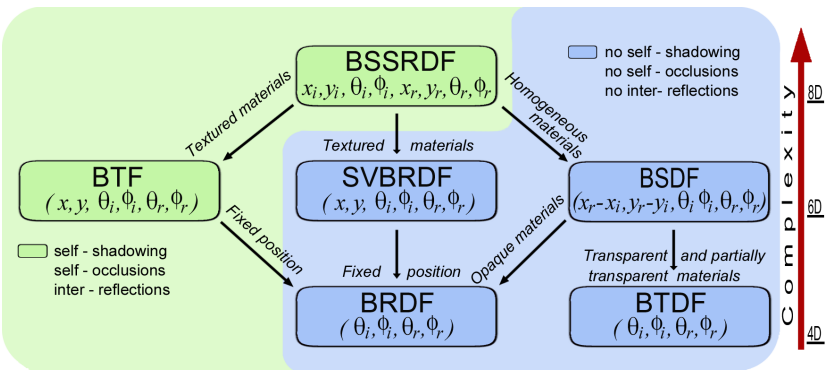

Figure 3: Taxonomy of the selected reflectance functions derived as further simplifications of the BSSRDF, according to [MMS*04, WLL*09, DLG13, HF13].

rotated analytical BRDFs [WDR11]. The first BTF database, described in [DVGNK99], contains 61 real-world surfaces, each observed under 205 different combinations of lighting and viewing illuminations (plus 205 additional measurements for anisotropic surfaces), consists of over 14.000 images. For a recent survey on BTF measurement, modelling, and compression methods see [FH09].

For a homogeneous material, an intermediate representation between the BSSRDF and the BRDF, comprising scattering effects for both reflection and transmission, is given by the Bidirectional Scattering Distribution Function (BSDF). The concept of BSDF is generally understood as a sum of a BRDF and a Bidirectional Transmittance Distribution Fuction (BTDF), the latter modelling how the light passes through a (semi)transparent surface [WMLT07, HF13](see Figure 1(c)). The taxonomy of the reflectance functions introduced in this Section, showing their relationships with the BRDF along with their parameterisation, is reported in Figure 3: each of them can be considered as a special case of the BRDF [ASMS01].

\subsection{Properties of the BRDF}

There are many reflectance models that are simplified subsets of the BRDF function. One of the simplest reflectance models is the Lambertian model, which represents the perfect diffuse reflectance and is often used in many interactive applications, since it requires no recalculation with the change of viewing direction. The model simply assumes that the surface reflects light uniformly in all directions with the same radiance (see Figure 4(a), in light blue, and Figure 4(b)), constant with $v_{r}$ unlike other BRDF models: $f_{r}\left(\mathbf{v}_{\mathbf{i}}, \mathbf{v}_{\mathbf{r}}\right)=\rho_{d} / \pi$, where $\rho_{d}$ is the diffuse albedo.

In the case of a pure specular BRDF all the light is reflected in a single direction, for a given incident direction (see Figure 4(a), in blue, and Figure 4(d)). In fact, light that is incident within a differential solid angle $d \omega_{i}$ from direction $\left(\theta_{i}, \phi_{i}\right)$ is reflected in a differential solid angle $\omega_{r}$ in direction $\left(\theta_{i}, \phi_{i}+\pi\right)$, hence the pure specular BRDF can be formalised with a double Dirac delta function: $f_{r}\left(\mathbf{v}_{\mathbf{i}}, \mathbf{v}_{\mathbf{r}}\right)=\rho_{s} \delta\left(\theta_{i}-\theta_{r}\right) \delta\left(\phi_{i}+\pi-\phi_{r}\right)$, where $\rho_{s}=L_{r} / L_{i}$ is the specular albedo. Perfect specularity is valid only for highly polished mirrors and metals.

Surfaces not perfectly smooth, which have some roughness at the micro-geometry level, have a glossy appearance and show broader

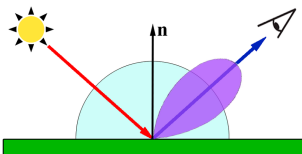

(a) Basic reflection models

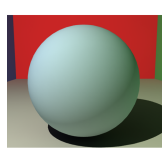

(b) Diffuse

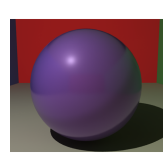

(c) Glossy

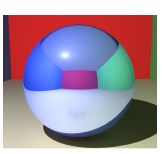

(d) Specular
Figure 4: (a) Basic reflectance models of the incoming light (in red): perfect diffuse (light blue), glossy (purple) and perfect specular (blue). In (b-d) renderings of diffuse, glossy and specular spheres are shown, placed inside a Cornell box [GTGB84].

highlights, other than specular reflections (see Figure 4(a), in purple, and Figure 4(c)).

Some materials, like the surface of the moon or some biological tissues, show a phenomenon called retro-reflection in which light is scattered not only in the forward direction but also in the direction of the illuminant.

A BRDF should respect some basic physical properties, namely non-negativity, reciprocity and energy conservation:

- non-negativity: the BRDF is a non-negative function, hence for any pair of incident and outgoing direction $f_{r}\left(\mathbf{v}_{\mathbf{r}}, \mathbf{v}_{\mathbf{i}}\right) \geq 0$;

- the Helmholtz reciprocity principle states that the light path is reversible, for any pair of incident and outgoing direction: $f_{r}\left(\mathbf{v}_{\mathbf{i}}, \mathbf{v}_{\mathbf{r}}\right)=f_{r}\left(\mathbf{v}_{\mathbf{r}}, \mathbf{v}_{\mathbf{i}}\right)$. This principle holds only for corresponding states of polarisation for incident and emerging fluxes, whereas large discrepancies might occur for non-corresponding states of polarisation [CP85]. In designing a rendering system possible non-reciprocity should be taken into account [Vea97].

- Energy conservation assumes that the energy reflected cannot exceed incident energy [DRS07]: $L_{r} \leq E_{i}$ hence over the unit hemisphere $\Omega_{+}$above the surface

$$
\forall \mathbf{v}_{\mathbf{i}}, \int_{\Omega_{+}} f_{r}\left(\mathbf{v}_{\mathbf{i}}, \mathbf{v}_{\mathbf{r}}\right)\left(\mathbf{v}_{\mathbf{r}} \cdot \mathbf{n}\right) d \omega_{r} \leq 1
$$

BRDFs can be classified by taking into account the characteristics of the material to represent:

- Isotropic BRDFs are able to represent materials whose reflection does not depend on the orientation of the surface, since the reflectance properties are invariant to rotations of the surface around $\mathbf{n}$.

- Anisotropic BRDFs can describe materials whose reflection change with respect to rotation of the surface around $\mathbf{n}$; this class includes materials like brushed metal, satin, velvet and hair.

The Fresnel effect predicts the fraction of power which is reflected and transmitted and has a great impact on the appearance. Many basic BRDF models have lost importance in the context of physically based modelling because they do not account for a Fresnel term. For conductive materials, like metals, the fraction of light reflected by pure specular reflection is roughly constant for all angles of incidence, whereas for non-conductive materials (dielectrics), the amount of light reflected increases at grazing angles; see Figure 5 for a comparative example of the behaviour of metals and dielectrics. The fraction of light reflected is called Fresnel reflectance, which can be obtained from the solution of Maxwell's 


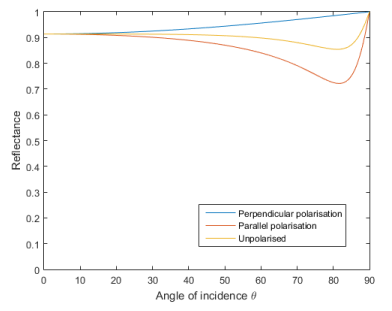

(a) Metal (aluminium)

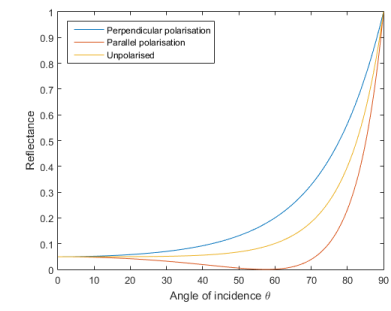

(b) Dielectric (plastic)
Figure 5: Fresnel reflectance for metals (a) and dielectrics $(b)$.

equations and depends also on the polarisation state of the incident light. For unpolarised light, the Fresnel reflectance $\Im$ at the interface between the surface and the air is given by

$\Im\left(\eta, \theta_{i}, \theta_{t}\right)=\frac{1}{2}\left[\left(\frac{\eta \cos \theta_{i}-\cos \theta_{t}}{\eta \cos \theta_{i}+\cos \theta_{t}}\right)^{2}+\left(\frac{\cos \theta_{i}-\eta \cos \theta_{t}}{\cos \theta_{i}+\cos \eta \theta_{t}}\right)^{2}\right]$,

where $\eta$ is the index of refraction of the surface and $\theta_{t}$ is the angle of transmission. In Computer Graphics, it is very common to use Schlick's approximation of the Fresnel reflectance [Sch94]: $\Im(\theta)=$ $\Im(0)+(1-\Im(0))(1-\cos (\theta))^{5}$; in Section 3 we will generally use the symbol $\Im$ to refer either to the exact Fresnel reflectance or one of its approximations.

\section{Models of BRDF}

Many BRDF models have been proposed for general material representation but in general they still require further development as the parameters are not intuitive and controllable enough for users [MU12].

Phenomenological models are entirely based on reflectance data, which is fitted to analytical formulas, thus approximating the reflectance and reproducing characteristics of real world materials. Some of the most important phenomenological models are described in Section 3.1.

Physically-based models, reported in Section 3.2, are based on Physics and Optics with the assumption that the surface is rough at a fine scale, therefore described by a collection of micro facets with some distribution $D$ of size and direction. Usually they are represented by accurate and adjustable formulae, however the most common mathematical model has the form:

$$
f_{r}\left(\mathbf{v}_{\mathbf{i}}, \mathbf{v}_{\mathbf{r}}\right)=\frac{D \cdot G \cdot \Im}{4 \cos \theta_{i} \theta_{r}}
$$

which also takes into account the Fresnel term $\Im$. Effect like masking and self-shadowing (see Figure 6) [AMHH08] depend on the projected area of the microfacets and hence on the distribution $D$, generally described by the geometrical attenuation term $G$; for a review of common masking functions and a derivation of the exact form of the masking function from the microsurface profile, see the work by Heitz [Hei14]. This class of models can represent unique properties of the material and may include subsurface structure, generally resulting in complex calculations due to the interaction of the light with the surface structure.

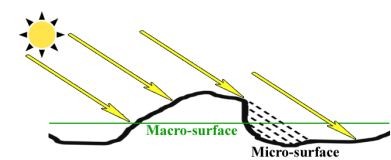

(a) Shadowing

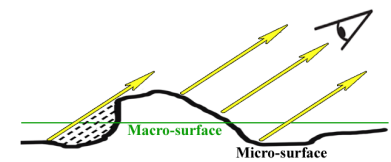

(b) Masking
Figure 6: (a) Due to the microgeometry, some microfacets are occluded and do not receive light (shadowing). (b) The light reflected from microfacets not visible from the viewing direction can not be seen (masking)

Measured BRDF data, produced by most of the setups described in Section 4, can be stored in a table or a grid and then interpolated, to produce a large look-up table when data is needed. This method is simple but inefficient in terms of storage. Moreover, the measured raw data is often noisy, hence the noise is likely to appear in the rendered material. A measured BRDF can be fitted to analytic models and employed to reconstruct the BRDF, thus significantly reducing storage size. The down side of this strategy is related to the inflexibility of many models, hard to edit and able to represent only limited classes of materials. A different solution is to to approximate measured BRDFs with a suitable function space, e.g. spherical harmonics or wavelets, weighted sum of separable functions or product of functions. We refer to this class of models as Data-Driven models, described in Section 3.3.

Many medium, such as hair, fur, cloth and knitwear are difficult to describe by a surface model. These materials, and objects with highly complex boundary, are better described by volumetric appearance models [KK89, PH89, XCL $\left.{ }^{*} 01\right]$, in particular for closer viewing distance, whereas BRDFs can be used from farther away. Jakob et al. [JAM $\left.{ }^{*} 10\right]$ introduced a generalisation to anisotropic scattering structures, exploited also for volumes acquired by CT scans [ZJMB11]. More recently, collections of individual fibers have been used for fabric representation [KSZ* 15$]$. In this paper we focus on representation and acquisition of surface reflectance, hence we do not further discuss volumetric representation.

An important aspect is the practicality of a model in a rendering system, which requires a suitable technique for importance sampling. When calculating the radiance direction of a surface in a scene, accounting for the contribution of light from all possible directions is expensive to compute, therefore Monte Carlo techniques are used to estimate the values with fewer samples [Hai91], based on a stochastic process. However, the number of samples should be sufficient to produce consistent estimations, otherwise the results will vary significantly. Importance sampling can be used to reduce sample variance [LRR04], by distributing samples according to the known elements, either taking into account the reflection model in use or the incident light [CJAMJ05].

Table 1 summarises the characteristics of the reflectance models detailed in the following sections, selected to widely cover as many possible unique models proposed in the Computer Graphics and Vision literature, suitable for a broad range of materials. 


\begin{tabular}{|c|c|c|c|c|c|c|}
\hline & Model and Ref & 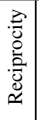 & 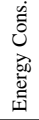 & 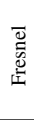 & 竞 & Short Description \\
\hline \multicolumn{7}{|c|}{ PHENOMENOLOGICAL } \\
\hline \multirow{4}{*}{ 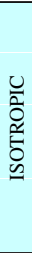 } & Phong [Pho75] & $x$ & $x$ & $x$ & $\checkmark$ & $\begin{array}{l}\text { Basic surface representation model } \\
\text { based on the Cosine law. }\end{array}$ \\
\hline & $\begin{array}{l}\text { Blinn-Phong } \\
\text { [Bli77] }\end{array}$ & $x$ & $x$ & $x$ & $\checkmark$ & $\begin{array}{l}\text { Based on [Pho75], uses the halfway re- } \\
\text { flection direction for faster computation. }\end{array}$ \\
\hline & $\begin{array}{l}\text { Nishino and } \\
\text { Lombardi [NL11] }\end{array}$ & $\checkmark$ & $\checkmark$ & $x$ & $x$ & $\begin{array}{l}\text { Models BRDFs as a mixture of hemi- } \\
\text { spherical distribution functions; small } \\
\text { footprint. }\end{array}$ \\
\hline & $\begin{array}{l}\text { Brady et al. } \\
\text { [BLPW14] }\end{array}$ & $\checkmark$ & $x$ & $\checkmark$ & $x$ & $\begin{array}{l}\text { Framework for automatic learning of an- } \\
\text { alytical models. Some of the properties } \\
\text { are not guaranteed by the grammar. }\end{array}$ \\
\hline \multirow{6}{*}{ 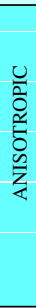 } & Ward [War92] & 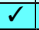 & $x$ & $x$ & $\checkmark$ & Versatile and cheap to compute. \\
\hline & $\begin{array}{l}\text { Lafortune } \\
\text { [LFTG97] }\end{array}$ & $\checkmark$ & $\checkmark$ & $x$ & $\checkmark$ & $\begin{array}{l}\text { Generalisation of the cosine lobe model } \\
\text { with multiple steerable lobes. }\end{array}$ \\
\hline & $\begin{array}{l}\text { Neumann et al. } \\
\text { [NNSK99] }\end{array}$ & $\checkmark$ & $\checkmark$ & $\checkmark$ & $\checkmark$ & $\begin{array}{l}\text { Physically plausible formulation of the } \\
\text { Phong, Blinn-Phong and Ward models. }\end{array}$ \\
\hline & $\begin{array}{l}\text { Ashikhmin- } \\
\text { Shirley [AS00] }\end{array}$ & $x$ & $x$ & $\checkmark$ & $\checkmark$ & $\begin{array}{l}\text { Based on [Pho75], includes anisotropic } \\
\text { reflections for two-layered materials. }\end{array}$ \\
\hline & $\begin{array}{l}\text { Edwards et al. } \\
{\left[\mathrm{EBJ}^{*} 06\right]}\end{array}$ & $x$ & $\checkmark$ & $x$ & $\checkmark$ & $\begin{array}{l}\text { Framework for transforming the halfway } \\
\text { vector into different domains. }\end{array}$ \\
\hline & $\begin{array}{l}\text { Ashikhmin- } \\
\text { Premoze [AP07] }\end{array}$ & $\checkmark$ & $\checkmark$ & $\checkmark$ & $\checkmark$ & $\begin{array}{l}\text { Combines analytic model with a } \\
\text { data-driven distribution; accounts for } \\
\text { backscattering. }\end{array}$ \\
\hline \multicolumn{7}{|c|}{ PHYSICALLY-BASED } \\
\hline \multirow{10}{*}{ 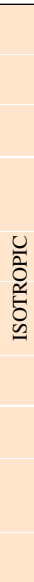 } & $\begin{array}{l}\text { Cook-Torrance } \\
\text { [CT82] }\end{array}$ & $\checkmark$ & $x$ & $\checkmark$ & $x$ & $\begin{array}{l}\text { It can model metals and plastics, view } \\
\text { dependent changes. }\end{array}$ \\
\hline & $\begin{array}{l}\text { He et al. } \\
\text { [HTSG91] }\end{array}$ & $\checkmark$ & $\checkmark$ & $\checkmark$ & $x$ & $\begin{array}{l}\text { Enhances [CT82], allowing more gen- } \\
\text { eral material representation. }\end{array}$ \\
\hline & $\begin{array}{l}\text { Oren-Nayar } \\
\text { [ON94] }\end{array}$ & $\checkmark$ & $\checkmark$ & $x$ & $\checkmark$ & $\begin{array}{l}\text { Enhance the Lambertian model for } \\
\text { rough diffuse surfaces. }\end{array}$ \\
\hline & $\begin{array}{l}\text { Ershov et al. } \\
\text { [EKM01] }\end{array}$ & $x$ & $\checkmark$ & $\checkmark$ & $x$ & $\begin{array}{l}\text { Focuses on layered materials, like metal- } \\
\text { lic paint. It models binder pigment parti- } \\
\text { cles, flakes and flake coating. }\end{array}$ \\
\hline & $\begin{array}{l}\text { Walter et al. } \\
\text { [WMLT07] }\end{array}$ & $\checkmark$ & $\checkmark$ & $\checkmark$ & $\checkmark$ & $\begin{array}{l}\text { Defines the GGX distribution; based on } \\
\text { a BSDF representation. }\end{array}$ \\
\hline & $\begin{array}{l}\text { Rump et al. } \\
\text { [RMS*08] }\end{array}$ & $x$ & $x$ & $\checkmark$ & $\checkmark$ & $\begin{array}{l}\text { Suitable for metallic paints, combines } \\
\text { [CT82] for the base layer with BTF for } \\
\text { top paint layer, including particles. }\end{array}$ \\
\hline & $\begin{array}{l}\text { Kurt et al. } \\
\text { [KSKK10] }\end{array}$ & $\checkmark$ & $\checkmark$ & $\checkmark$ & $\checkmark$ & $\begin{array}{l}\text { The multiple specular lobe model can } \\
\text { represent layered or mixed materials. }\end{array}$ \\
\hline & $\begin{array}{l}\text { Bagher et al. } \\
\text { [BSH12] }\end{array}$ & $\checkmark$ & $x$ & $\checkmark$ & $\checkmark$ & $\begin{array}{l}\text { Provides accurate fitting for materials in } \\
\text { the MERL database }\end{array}$ \\
\hline & $\begin{array}{l}\text { Löw et al. } \\
\text { [LKYU12] }\end{array}$ & $\checkmark$ & $x$ & $\checkmark$ & $\checkmark$ & $\begin{array}{l}\text { Guarantees accurate fitting to measured } \\
\text { data for glossy surfaces; descibes } 2 \text { mod- } \\
\text { els based on the ABC distribution }\end{array}$ \\
\hline & $\begin{array}{l}\text { Jakob et al. } \\
{\left[\mathrm{JHY}^{*} 14\right]}\end{array}$ & $x$ & $x$ & $\checkmark$ & $\checkmark$ & $\begin{array}{l}\text { Allows modelling spatially varying } \\
\text { BRDF appearance of glittery surfaces }\end{array}$ \\
\hline \multirow{2}{*}{$\sum_{<}^{\dot{n}}$} & $\begin{array}{l}\text { Weidlich and } \\
\text { Wilkie [WW07] }\end{array}$ & $\checkmark$ & $\checkmark$ & $\checkmark$ & $\checkmark$ & $\begin{array}{l}\text { Multi-layered model which includes ab- } \\
\text { sorbtion and internal reflection. }\end{array}$ \\
\hline & $\begin{array}{l}\text { Dupuy et al. } \\
\text { [DHI*15] }\end{array}$ & $x$ & $x$ & $\checkmark$ & $\checkmark$ & $\begin{array}{l}\text { Method to automatically convert a mate- } \\
\text { rial to a microfacet BRDF. }\end{array}$ \\
\hline \multicolumn{7}{|c|}{ DATA-DRIVEN } \\
\hline \multirow{3}{*}{ 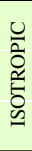 } & $\begin{array}{l}\text { Matusik et al. } \\
\text { [MPBM03a] }\end{array}$ & $\checkmark$ & $\checkmark$ & $x$ & $\checkmark$ & $\begin{array}{l}\text { Provides realistic appearance and mean- } \\
\text { ingful parameterisation }\end{array}$ \\
\hline & $\begin{array}{l}\text { Matusik et al. } \\
\text { [MPBM03b] }\end{array}$ & $\checkmark$ & $x$ & $x$ & $\checkmark$ & $\begin{array}{l}\text { Reduces number of samples to acquire } \\
\text { and represent BRDF }\end{array}$ \\
\hline & $\begin{array}{l}\text { Romeiro et al. } \\
{[\text { RVZ08] }}\end{array}$ & $\checkmark$ & $x$ & $\checkmark$ & $\checkmark$ & $\begin{array}{l}\text { Bivariate representation, allows to cap- } \\
\text { ture off-specular and retro-reflections. }\end{array}$ \\
\hline \multirow{8}{*}{ 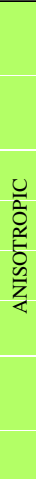 } & $\begin{array}{l}\text { Kautz and McCool } \\
\text { [KM99] }\end{array}$ & $x$ & $x$ & $x$ & $\checkmark$ & $\begin{array}{l}\text { SVD or ND based based decomposition } \\
\text { for BRDFs; approximation based on tex- } \\
\text { tures, used to store directions. }\end{array}$ \\
\hline & $\begin{array}{l}\text { McCool and } \\
\text { Ahmad [MAA01] }\end{array}$ & $\checkmark$ & $\checkmark$ & $x$ & $\checkmark$ & $\begin{array}{l}\text { Based on logarithmic homomorphism. } \\
\text { Simple parameterisation and limited } \\
\text { storage cost ( } 2 \text { textures). }\end{array}$ \\
\hline & $\begin{array}{l}\text { Lawrence et al. } \\
\text { [LRR04] }\end{array}$ & $x$ & $x$ & $x$ & $\checkmark$ & $\begin{array}{l}\text { Provides accurate results and can be also } \\
\text { used for BTFs; compact representation }\end{array}$ \\
\hline & $\begin{array}{l}\text { Lawrence et al. } \\
{\left[\text { LBAD* 06] }^{*}\right.}\end{array}$ & $\checkmark$ & $\checkmark$ & $\checkmark$ & $x$ & Suited for interactive rendering/editing \\
\hline & $\begin{array}{l}\text { Ozturk et al. } \\
\text { [OKBG08] }\end{array}$ & $\checkmark$ & $x$ & $x$ & $x$ & $\begin{array}{l}\text { Computationally efficient linear model } \\
\text { for approximating BRDFs. }\end{array}$ \\
\hline & $\begin{array}{l}\text { Bilgili et al. } \\
\text { [BÖK11] }\end{array}$ & $x$ & $x$ & $\checkmark$ & $\checkmark$ & $\begin{array}{l}\text { Recursive application of the Tucker de- } \\
\text { composition on the error term }\end{array}$ \\
\hline & $\begin{array}{l}\text { Pacanowski et } \\
\text { al. [PSCS* 12] }\end{array}$ & $\checkmark$ & $x$ & $\checkmark$ & $\checkmark$ & $\begin{array}{l}\text { Projects measured BRDFs on a 2D space } \\
\text { and approximates them with Rational } \\
\text { Functions; small footprint. }\end{array}$ \\
\hline & $\begin{array}{l}\text { Ward et al. } \\
\text { [WKB14] }\end{array}$ & $\checkmark$ & $x$ & $x$ & $\checkmark$ & $\begin{array}{l}\text { Tensor tree representation for measured } \\
\text { BSDF data. }\end{array}$ \\
\hline
\end{tabular}

Table 1: Some of the main properties of the BRDF models described in Section 3. Models are grouped by category; within each isotropic/ anisotropic sub-category models are sorted by year.

\subsection{Phenomenological models}

One of the earliest models for non-Lambertian surfaces is the Phong [Pho75] model, which is based on the cosine law. It is a computationally convenient method for analytically approximating the reflectance properties of a set of materials with a slightly rough surface: $f_{r}\left(\mathbf{v}_{\mathbf{i}}, \mathbf{v}_{\mathbf{r}}\right)=k_{s}\left(\mathbf{v}_{\mathbf{r}} \cdot \mathbf{r}_{\mathbf{v} \mathbf{i}}\right)^{n}$, where $k_{s}$ is a specular constant in the range $[0, \infty], \mathbf{r}_{\mathbf{v i}}$ is the direction of $\mathbf{v}_{\mathbf{i}}$ after being perfectly reflected and $n$ controls the shape of the specular highlight. This model simulates the effects of both specular and diffuse reflections, but does not take into account energy conservation nor reciprocity. Moreover, it does not capture the reflection behaviour of real surfaces at grazing angles. By being very simple to compute, it became commonly used in Computer Graphics to describe peaks of reflection [War92]. Since it is not normalised, some normalisation factors for cosine lobes have been proposed, either based on double-axis moments [Arv95] or with the simpler option of a power series in $(\mathbf{n} \cdot \mathbf{h})$ with a suitable sequence of exponents [Lew94].

The Blinn-Phong reflection model [Bli77] enhances the Phong model, since it uses the halfway vector $\mathbf{h}$ and the normal $\mathbf{n}$ instead of the reflection vector $\mathbf{r}_{\mathbf{v i}}$, thus reducing the computational cost associated with the need to constantly calculate the latter vector:

$$
f_{r}\left(\mathbf{v}_{\mathbf{i}}, \mathbf{v}_{\mathbf{r}}\right)=k_{S}(\mathbf{n} \cdot \mathbf{h})^{n}
$$

Although it has been used as the default shading model for OpenGL and Direct3D until recent times, it shares the same limitations of the Phong model, being physically not plausible and not able to capture metallic and mirrored appearance. Since it follows the cosine function, if $n$ goes to infinity the reflected radiance and the albedo converges to zero towards grazing angles.

The Ward reflectance model [War92] was specifically designed to easily fit measured BRDFs, which have been used for validation. The model is able to represent both isotropic and anisotropic reflection; it combines specular and diffuse components of reflectance, representing specular peaks through Gaussian distributions. The model specifies a normalisation factor which allows a correct integration over the hemisphere and it is very efficient for Monte Carlo sampling. The Ward model has four parameters, which can be set independently, therefore it can be fitted to a large class of measured data. The Ward isotropic model is given by:

$$
f_{r}\left(\mathbf{v}_{\mathbf{i}}, \mathbf{v}_{\mathbf{r}}\right)=\frac{\rho_{d}}{\pi}+\frac{\rho_{s}}{\sqrt{\cos \left(\theta_{i}\right) \cos \left(\theta_{r}\right)}} \cdot \frac{e^{-\tan ^{2}\left(\frac{\theta_{h}}{\alpha^{2}}\right)}}{4 \pi \alpha^{2}}
$$

where $\rho_{s}$ controls the magnitude of the lobe, $\alpha$ controls the width of the Gaussian lobe and $4 \pi \alpha^{2}$ is a normalisation factor. The anisotropic model makes use of the two parameters $\alpha_{x}$ and $\alpha_{y}$ to control the width of the gaussian lobe in the two principal directions of anisotropy:

$$
f_{r}\left(\mathbf{v}_{\mathbf{i}}, \mathbf{v}_{\mathbf{r}}\right)=\frac{\rho_{d}}{\pi}+\frac{\rho_{s}}{\sqrt{\cos \left(\theta_{i}\right) \cos \left(\theta_{r}\right)}} \cdot \frac{e^{-\tan ^{2}\left(\theta_{h}\right)\left(\frac{\cos ^{2} \theta_{h}}{\alpha_{x}^{2}}+\frac{\sin ^{2} \theta_{h}}{\alpha_{y}^{2}}\right)}}{4 \pi \alpha_{x} \alpha_{y}}
$$

The model does not obey the principle of energy conservation at grazing angles, which has been investigated in [NNSK99, Dür06, GMD10]. A different normalisation factor has been proposed in [Dür06] to prevent numerical instabilities and to correct the loss 
of energy at flat angles, specifically $\left(4 \cos \left(\theta_{i}\right) \cos \left(\theta_{r}\right)\right)$ instead of $\left(4 \sqrt{\cos \left(\theta_{i}\right) \cos \left(\theta_{r}\right)}\right)$, however it shares the problem of diverging to infinity with the original Ward model. A new physically plausible version of the model has been proposed in [GMD10], which meets the energy conservation principle even at grazing angles by using the following normalisation factor:

$$
\frac{2\left(1+\cos \theta_{i} \cos \theta_{r}+\sin \theta_{i} \sin \theta_{r} \cos \phi_{r}-\phi_{r}\right)}{\left(\cos \theta_{i} \cos \theta_{r}\right)^{4}} .
$$

Neumann et al. [NNSK99] proposed some modifications and correction factors for the reciprocal Phong [Pho75, LW94], Blinn [Bli77] and Ward [War92] models. The correction factors can be seen as shadowing and masking terms to make the models physically plausible. Moreover the modified models can be used to render metals and other specular objects and for each of them an importance sampling procedure is described.

The Lafortune [LFTG97] model is a flexible, empirical model designed to fit measurements from real surfaces and compactly represent them [WLT04]. The model is a generalisation of the cosine lobe model with multiple steerable lobes, based on the Phong shading model. The primitive functions obey the Energy Conservation and Reciprocity principles. This model allows lobe specification on the surface in terms of shape and direction, by simply setting up to 3 parameters and an exponent :

$$
f_{r}\left(\mathbf{v}_{\mathbf{i}}, \mathbf{v}_{\mathbf{r}}\right)=\frac{\rho_{d}}{\pi}+\sum_{l=1}^{N}\left(C_{x, l} v_{i x} v_{r x}+C_{y, l} v_{i y} v_{r y}+C_{z, l} v_{i z} v_{r z}\right)^{n_{l}}
$$

where $N$ is the number of lobes, $C_{x}, C_{y}, C_{z}$ are parameters which absorb the specular albedo and control retro-reflections (by setting $C_{x}, C_{y}$ and $C_{z}$ to positive values), anisotropy (with $C_{x} \neq C_{y}$ ) and off-specular peaks (if $C_{z}$ is smaller than $-C_{x}=-C_{y}$ ). Lafortune's reflection model can represent generalised diffuse reflectance as the model is able to reflect radiance evenly in all directions, by setting $C_{x}=C_{y}=0$; the Lambertian model can be obtained by setting $N=0$. A comparative study shows that the Lafortune model performs better than the Phong, Ward and He et al. models in representing measured BRDFs like white paper, rough plastic, rough aluminium and metal, since it was designed to fit almost any BRDF data [WLT04].

The Ashikhmin-Shirley model is based on the Phong reflectance model and describes both diffuse and specular reflections [AS00]. The reflectance of the model changes with the view-point, hence at grazing angles the reflectance is specular and at normal angles the reflectance is diffuse. This model assumes micro facets with various angles and sizes [OKBG08] by generalising the types of microfacets and allows the expression of arbitrary angles. The specular component of the BRDF is expressed by:

$$
f_{r, s}\left(\mathbf{v}_{\mathbf{i}}, \mathbf{v}_{\mathbf{r}}\right)=\frac{\Im\left(\mathbf{v}_{\mathbf{i}} \cdot \mathbf{h}\right) D(\mathbf{h})}{2\left(\mathbf{h} \cdot \mathbf{v}_{\mathbf{r}}\right) \max \left(\mathbf{n} \cdot \mathbf{v}_{\mathbf{r}}, \mathbf{n} \cdot \mathbf{v}_{\mathbf{i}}\right)}
$$

where $\Im\left(\mathbf{v}_{\mathbf{i}} \cdot \mathbf{h}\right)$ is the Schlick's approximation of the Fresnel term [Sch94]. $D(\mathbf{h})$ is the distribution function of the microfacets, controlled by the parameters $e_{x}$ and $e_{y}$, the axes of an ellipse which orientates the halfway vector $\mathbf{h}$ of the microfacets respectively along the $X$ and $Y$ and thus defining the anisotropy:

$$
D(\mathbf{h})=\frac{\sqrt{\left(e_{x}+1\right)\left(e_{y}+1\right)}(\mathbf{h} \cdot \mathbf{n})^{e_{x} \cos ^{2}\left(\phi_{h}\right)+e_{y} \sin ^{2}\left(\phi_{h}\right)}}{4 \pi} .
$$

In order to preserve energy conservation and to model the behaviour of the surface's diffuse colour near the grazing angle, which disappears due to the increase in specular reflectance, instead of a Lambertian diffuse term an angle-dependent form of the diffuse component is reported. The expression is based on the consideration that the amount of energy for diffuse scattering is dependent on the total reflectance of the specular term at the incident angle:

$$
f_{r, d}\left(\mathbf{v}_{\mathbf{i}}, \mathbf{v}_{\mathbf{r}}\right)=\left(1-\rho_{s}\right) g\left(\mathbf{v}_{\mathbf{i}}, \mathbf{v}_{\mathbf{r}}, \mathbf{n}\right)\left(28 \rho_{d}\right) /(23 \pi)
$$

where $g\left(\mathbf{v}_{\mathbf{i}}, \mathbf{v}_{\mathbf{r}}, \mathbf{n}\right)=\left[1-\left(1-\left(\mathbf{n} \cdot \mathbf{v}_{\mathbf{i}}\right) / 2\right)^{5}\right]\left[1-\left(1-\left(\mathbf{n} \cdot \mathbf{v}_{\mathbf{r}}\right) / 2\right)^{5}\right]$. The model is able to describe anisotropic reflections of two layered materials, such as varnished wood for example, it is physically plausible and controlled by simple and intuitive parameters. A sampling method for Monte Carlo rendering is also provided, based on $D(\mathbf{h})$ : it gives the probability density function $p\left(\mathbf{v}_{\mathbf{r}}\right)=D(\mathbf{h}) / 4\left(\mathbf{v}_{\mathbf{i}} \cdot \mathbf{h}\right)$.

The Ashikhmin-Premoze model [AP07], or d-BRDF, follows [TS67] and [CT82] microfacet theory and it is based on the earlier [AS00] model, with a simplified process of fitting BRDF models to measured data; an efficient sampling technique is also suggested. The Ashikhmin-Premoze model combines an analytic model with a data driven distribution and also discusses how to fit backscattering measurements to the model [GHP*08]. The model allows the use of an arbitrary normalised function $p(\mathbf{h})$, instead of restricting the shape of the distribution to specific shapes such as Phong or Gaussian function which simplifies mathematical formulation. Specular highlights can be easily adjusted since their shape depends directly on the distribution. The $\max$ term in Equation 10, which causes colour banding artifacts as observed in [AP07], is replaced with a smoother term $\left(\mathbf{v}_{\mathbf{i}} \cdot \mathbf{n}\right)+\left(\mathbf{v}_{\mathbf{r}} \cdot \mathbf{n}\right)-\left(\mathbf{v}_{\mathbf{i}} \cdot \mathbf{n}\right)\left(\mathbf{v}_{\mathbf{r}} \cdot \mathbf{n}\right)$. An additional modification is to exclude the $\left(\mathbf{h} \cdot \mathbf{v}_{\mathbf{r}}\right)$ term to improve the appearance matching with real world materials. The resulting expression for the specular term, which is reciprocal and non negative for any non-negative $p(\mathbf{h})$, can be written as:

$$
f_{r, s}\left(\mathbf{v}_{\mathbf{i}}, \mathbf{v}_{\mathbf{r}}\right)=\frac{\Im\left(\mathbf{v}_{\mathbf{i}} \cdot \mathbf{h}\right) p(\mathbf{h}) k_{s}}{\left(\mathbf{v}_{\mathbf{i}} \cdot \mathbf{n}\right)+\left(\mathbf{v}_{\mathbf{r}} \cdot \mathbf{n}\right)-\left(\mathbf{v}_{\mathbf{i}} \cdot \mathbf{n}\right)\left(\mathbf{v}_{\mathbf{r}} \cdot \mathbf{n}\right)}
$$

where $k_{s}$ is a scaling constant which needs to be chosen in order to fulfill energy conservation. The d-BRDF model improves representation of material reflectance at grazing angles and enables more realistic material appearance, however some effects like retroreflection cannot be modeled properly. Moreover, the Fresnel parameters do not have a physical meaning, since the optimal values can lie significantly outside of the real range for a given material.

Edwards et al. proposed a framework for transforming the halfway vector $\mathbf{h}$ into different domains to enforce energy conservation but compromising reciprocity [EBJ*06]. By writing Equation 2 in terms of $\forall \mathbf{v}_{\mathbf{r}}$ and assuming that it satisfies an equality instead of an inequality, the function

$$
Q\left(\mathbf{v}_{\mathbf{i}}\right)=f_{r}\left(\mathbf{v}_{\mathbf{i}}, \mathbf{v}_{\mathbf{r}}\right)\left(\mathbf{v}_{\mathbf{i}} \cdot \mathbf{n}\right)
$$

can be seen as a probability density function (PDF) over the set of incident directions $\mathbf{v}_{\mathbf{i}}$ on the hemisphere $\Omega_{+}$. Since the PDF 
$Q\left(\mathbf{v}_{\mathbf{i}}\right)$ is related to a PDF $q(\mathbf{h})$ over halfway vectors by the formula $Q\left(\mathbf{v}_{\mathbf{i}}\right)=q(\mathbf{h}) /\left(4 \mathbf{v}_{\mathbf{i}} \cdot \mathbf{h}\right)$, from Equation 14 the following expression for $f_{r}\left(\mathbf{v}_{\mathbf{i}}, \mathbf{v}_{\mathbf{r}}\right)$, which conserves energy, can be derived:

$$
f_{r}\left(\mathbf{v}_{\mathbf{i}}, \mathbf{v}_{\mathbf{r}}\right)=[q(\mathbf{h})] /\left[4\left(\mathbf{v}_{\mathbf{i}} \cdot \mathbf{n}\right)\left(\mathbf{v}_{\mathbf{i}} \cdot \mathbf{h}\right)\right] .
$$

In the space of incident directions $\mathbf{v}_{\mathbf{i}}$ it is difficult to formulate a PDF to describe off-specular reflection and other phenomena. As for the halfway vector domain, near to grazing angles the set of allowable halfway vectors changes in a complicated way. If a new domain $D_{h}$ is defined, together with a PDF $p(\mathbf{l})$ and a bijection $f(\mathbf{h})=\mathbf{l}$ between the set of halfway vectors $\mathbf{h} \in \Omega_{+}$and the set of points $\mathbf{I} \in D_{h}$, by equating the differential probabilities between $D_{h}$ and $\Omega_{+}$the following can be derived from Equation 15 :

$$
f_{r}\left(\mathbf{v}_{\mathbf{i}}, \mathbf{v}_{\mathbf{r}}\right)=[p(\mathbf{l}) d \mu(\mathbf{I})] /\left[\left(4 \mathbf{v}_{\mathbf{i}} \cdot \mathbf{h}\right) d \omega_{h}\right]
$$

where $d \mu$ is the differential measure over $D_{h}$ and $p(\mathbf{l}) d \mu(\mathbf{l})=$ $q(\mathbf{h}) d \omega_{h}$. With this framework, a new domain can be defined given $\mathbf{v}_{\mathbf{r}}$, by translating $\Omega_{+}$so that the center of its base lies at the tip of $\mathbf{v}_{\mathbf{r}}$. In this way, every point on the translated hemisphere corresponds to an unnormalised halfway vector $\mathbf{h}_{\mathbf{u}}=\mathbf{v}_{\mathbf{i}}+\mathbf{v}_{\mathbf{r}}$. The final step is the transformation of the vectors $\mathbf{h}_{\mathbf{u}}$ to points $\mathbf{l}$ on the base of the hemisphere; if the local orientation of the surface is given by the orthogonal vectors $\mathbf{u}$ and $\mathbf{v}$, a point on the disk can be defined by the $(u, v)$ coordinates, hence the PDF $p(\mathbf{I})$ is two dimensional. A possibility is to scale the halfway vector until its tip lies in the base of the hemisphere, and the resulting energy conserving BRDF is:

$$
f_{r}\left(\mathbf{v}_{\mathbf{i}}, \mathbf{v}_{\mathbf{r}}\right)=\frac{p(\mathbf{I})\left(\mathbf{v}_{\mathbf{r}} \cdot \mathbf{n}\right)^{2}}{4\left(\mathbf{v}_{\mathbf{i}} \cdot \mathbf{n}\right)\left(\mathbf{v}_{\mathbf{i}} \cdot \mathbf{h}\right)(\mathbf{h} \cdot \mathbf{n})^{3}}
$$

since $\mathbf{l}=\frac{\left(\mathbf{v}_{\mathbf{r}} \cdot \mathbf{n}\right)}{\left(\mathbf{h}_{\mathbf{u}} \cdot \mathbf{n}\right)} \mathbf{h}_{\mathbf{u}}$. This transform allows to describe retroreflective materials by defining a PDF with high values near the center of the disk, which corresponds to a halfway vector in the retro-reflective direction; to specify a shiny BRDFs it is enough to define a PDF with high values near the origin of the $(u, v)$ space, which corresponds to $\mathbf{n}$ and gives pure specular reflection. To importance sample the BRDF to obtain $\mathbf{h}$ it is enough to generate a point $\mathbf{l}$ on the disk according to $p(\mathbf{l})$ and normalise. Alternatively, the orthogonal projection maps $\mathbf{h}_{\mathbf{u}}$ to the disk along the direction of $\mathbf{n}: \mathbf{l}=\mathbf{h}_{\mathbf{u}}-\left(\mathbf{v}_{\mathbf{i}} \cdot \mathbf{n}\right) \mathbf{n}$; it leads to a BRDF with narrower lobes, centered on the direction of perfect reflection. The resulting BRDF, suitable for data fitting, is given by:

$$
f_{r}\left(\mathbf{v}_{\mathbf{i}}, \mathbf{v}_{\mathbf{r}}\right)=\left[1 /\left(4\left(\mathbf{v}_{\mathbf{i}} \cdot \mathbf{h}\right)^{2}\right)\right] p(\mathbf{I})\left\|\mathbf{v}_{\mathbf{i}}+\mathbf{v}_{\mathbf{r}}\right\|^{2}
$$

To importance sample the BRDF, once a sample $\mathbf{l}$ is generated according to $p(\mathbf{I})$, the unnormalised halfway vector is obtained from the expression $\mathbf{h}_{\mathbf{u}}=\mathbf{l}+\left(\mathbf{v}_{\mathbf{i}} \cdot \mathbf{n}\right) \mathbf{n}$. Within the same framework two additional BRDF models are described: an empirical, energypreserving BRDF with limited number of parameters and a BRDF model useful for data fitting, which does not preserve energy.

Nishino and Lombardi [NL11] introduced a low-dimensional parametric BRDF model based on the idea of modeling BRDFs as a set of directional statistics distribution, able to encode many isotropic BRDFs with a small number of parameters. A BRDF is considered as a statistical distribution on a unit hemisphere, i.e. a probability density function that takes in input a direction for $\mathbf{v}_{\mathbf{i}}$ and returns a distribution of directions for $\mathbf{v}_{\mathbf{i}}$, called Hemispherical Exponential Power Distribution (Hemi-EPD). The Hemi-EPD constitutes a basis for the entire BRDF, which can be modeled as mixtures of Hemi-EPDs, one for each of its 2D slices. The expression for the Hemi-EPDs, given the parameters $\Theta=\kappa, \gamma$ and the normalization factor $C$, is $p\left(\theta_{h} \mid \theta_{d}, \Theta\right)=C(\Theta)\left(e^{\kappa \cos ^{\gamma} \theta_{h}}-1\right)$ and can represent a wide variety of distributions, including diffuse reflection. The optimal number $k$ of lobes is automatically determined by an Expectation-Maximisation algorithm, which tests different numbers of lobes with a statistical measure; as long as the condition $\sum_{1}^{k} 1 / C\left(\Theta_{k}\right) \leq 1$ holds, energy conservation is guaranteed. The $\mathrm{BRDF}$ is parameterised using the halfway vector and difference angle, by dropping the dependence on $\phi_{h}$ because of the isotropy.

Brady et al. [BLPW14] used Genetic Programming (GP) to develop a framework for learning new analytic BRDF models. GP is a machine learning technique which starts from a set of initial candidates and evolves them applying a set of pre-defined mutation and combinations, while trying to optimise a fitness function. A few basic BRDF models are used as a starting point (seeds), on which symbolic transformations are applied. The strategy used for the random search is heuristic-based, trying to adapt the starting models to some measured ground truth data of isotropic materials, and tends to produce a large set of candidates expressions. To allow a better exploration of the search space some suboptimal variations that increase the error are allowed and for the same purpose an island model genetic algorithm is used, allowing only sporadic interactions between sub-populations. The fitness function calculates the residual error of each variant after fitting the free parameters to the training set of materials, which consists of 8 materials from the MERL-MIT database [MPBM03a]. The grammar does not guarantee that the resulting models respect energy conservation and reciprocity, hence these properties need to be taken into account by the fitness function; in [BLPW14] is reported a table with some variants for which the properties have been numerically verified.

\subsection{Physically based models}

The Cook-Torrance model [CT82] became popular in computer graphics since it makes the distinction between metals and dielectrics, pays attention to microfacets, calculates the specular lobe using the halfway vector $\mathbf{h}$, and provides plausible results and orientation dependencies. The model takes into account both specular and diffuse reflections, the latter modeled as Lambertian reflections. As for the specular component, the model assumes that only the fraction of the facets oriented in the direction of $h$ contributes to the final reflection, moreover it accounts for how many facets are visible from different view angles and how they reflect light [WLL $\left.{ }^{*} 09\right]$. These factors are modeled respectively through the functions $D, G$ and $\Im$ :

$$
f_{r, s}\left(\mathbf{v}_{\mathbf{i}}, \mathbf{v}_{\mathbf{r}}\right)=\frac{\Im\left(\theta_{r}\right) D(\mathbf{h}) G\left(\mathbf{v}_{\mathbf{i}}, \mathbf{v}_{\mathbf{r}}\right)}{\pi \cos \left(\theta_{r}\right) \cos \left(\theta_{i}\right)} .
$$

The expression of the distribution $D(\mathbf{h})$ is generally a Gaussian: $D(\mathbf{h})=\cos \left(\theta_{r}\right) \exp ^{-\left(\frac{\alpha}{m}\right)^{2}}$, where $\alpha$ is the angle between $\mathbf{v}_{\mathbf{i}}$ and the reflected $\mathbf{v}_{\mathbf{r}}$ and $m$ is a roughness parameter. The attenuation term $G$ includes both the shadowing and masking effects:

$$
G\left(\mathbf{v}_{\mathbf{i}}, \mathbf{v}_{\mathbf{r}}\right)=\min \left(1, \frac{2(\mathbf{n} \cdot \mathbf{h})\left(\mathbf{n} \cdot \mathbf{v}_{\mathbf{r}}\right)}{\mathbf{v}_{\mathbf{r}} \cdot \mathbf{h}}, \frac{2(\mathbf{n} \cdot \mathbf{h})\left(\mathbf{n} \cdot \mathbf{v}_{\mathbf{i}}\right)}{\mathbf{v}_{\mathbf{r}} \cdot \mathbf{h}}\right) .
$$


One of the important contributions of this work is the formulation of the Fresnel term $\Im$, which represents the reflection of polished microfacets, approximated with the following expression:

$$
\Im(\theta)=\frac{(g-c)^{2}}{2(g+c)^{2}}\left(1+\frac{(c(g+c)-1)^{2}}{(c(g-c)+1)^{2}}\right)
$$

where $c=\mathbf{v}_{\mathbf{r}} \cdot \mathbf{h}$ and $g=\eta^{2}+c^{2}-1$, being $\eta$ the index of refraction. The Cook-Torrance model can properly model metals, plastic with varying roughness and view-dependent changes in colour, although it does not follow the energy conservation principle in the entire hemisphere; additional drawbacks are the not intuitive parameters.

Walter et al. [WMLT07] extend the microfacets theory introduced by [CT82] to simulate transmission through etched glass and other rough surfaces, thus taking into account the BSDF. The work by Smith [Smi67], which investigated the geometrical selfshadowing of a surface described by Gaussian statistics, is also extended by deriving a shadowing function from any microfacet distribution $D$; the BRDF component follows 4 . The distribution $D$ is different from previous models and has been developed to better fit measured data; it is named GGX and has the following expression:

$$
D(\mathbf{h})=\frac{\alpha_{g}^{2} \chi^{+}(\mathbf{h} \cdot \mathbf{n})}{\pi \cos ^{4} \theta_{h}\left(\alpha_{g}^{2}+\tan ^{2} \theta_{h}\right)^{2}}
$$

where $\alpha_{g}{ }^{2}$ is a width parameter and $\chi^{+}(x)$ is equal to one if $x>0$ and zero if $x \leq 0$. The GGX distribution has a stronger tail than previously used distributions, such as Beckmann and Phong, and thus tends to have more shadowing; in [BSH12] it has been observed that the GGX distribution is identical to the Trowbridge-Reitz distribution [TR75]. From $D$ it is possible to derive a simple sampling equation and the expression of $G$, which is given by:

$$
\begin{gathered}
G\left(\mathbf{v}_{\mathbf{i}}, \mathbf{v}_{\mathbf{r}}, \mathbf{h}\right) \approx G_{1}\left(\mathbf{v}_{\mathbf{i}}, \mathbf{h}\right) G_{1}\left(\mathbf{v}_{\mathbf{r}}, \mathbf{h}\right) \\
G_{1}\left(\mathbf{v}_{\mathbf{x}}, \mathbf{h}\right)=\chi^{+}\left(\frac{\mathbf{v}_{\mathbf{x}}, \mathbf{h}}{\mathbf{v}_{\mathbf{x}}, \mathbf{n}}\right) \frac{2}{1+\sqrt{1+\alpha_{g}^{2} \tan ^{2} \theta_{x}}} .
\end{gathered}
$$

The GGX distribution fails to properly capture the glowy highlights of highly polished surfaces like the chrome sample in the MERL database [MPBM03a], with a narrow specular peak and a much wider specular tail [MHH*12]. An anisotropic extension of the distribution, named Generalised-Trowbridge-Reitz, has been proposed by Burley [MHH* 12]; a symmetric extension of the GGX to the entire ellipsoid domain, suitable for volumetric anisotropic materials, is described by Heitz et al. [HDCD15].

Most of the materials in the MERL database [MPBM03a], like metals, metallic paints and shiny plastics, are very difficult to fit with commonly used distributions and generally require several lobes, due to the shape of the decrease in the BRDF, close to exponential at large angles but sharper at small angles. Bagher et al., based on this observation, suggested a function of $\tan ^{2} \theta_{h}^{-p}$ for the distribution $D$, where $p$ depends on the model [BSH12]. The model presented is the Cook-Torrance [CT82], in which the microfacets distribution is designed to efficiently and accurately approximate measured data. The distribution resulting from the suggested slope is called SGD (Shifted Gamma Distribution):

$$
D\left(\theta_{h}\right)=\frac{\chi_{[0, \pi / 2]}\left(\theta_{h}\right) \alpha^{p-1} e^{-\frac{\alpha^{2}+\tan ^{2} \theta_{h}}{\alpha}}}{\pi \cos ^{4} \theta_{h} \Gamma(1-p, \alpha)\left(\alpha^{2}+\tan ^{2} \theta_{h}\right)^{p}}
$$

where $\alpha$ is a fitting parameter, $\chi_{[0, \pi / 2]}\left(\theta_{h}\right)$ is is equal to 1 if $\theta_{h}<$ $\pi / 2$ and 0 otherwise, $\Gamma$ is the incomplete Gamma function:

$$
\Gamma(1-p, \alpha)=\int_{\alpha}^{\infty} t^{-p} e^{-t} d t .
$$

From the SGD it is possible to derive the shadowing function $G$ and a sampling method. An accurate fitting can be achieved for $97 \%$ of the materials in the MERL database with a single lobe, with the exeption of 3 multi-layered materials.

The He et al. model [HTSG91] extends previous optics models, including the geometric optics Cook-Torrance model [CT82], accounts for polarisation and masking/shadowing effects, includes specular reflection when the surface roughness is decreased and takes into account the nature of light as an electromagnetic wave, to model diffraction and interference. The model is able to represent metal, non-metal and plastic with smooth and rough surfaces and all parameters are physically based, thus resulting into a very complex BRDF model. The contribution to the reflection is given by three components, namely the specular term for mirror-like reflections, the directional diffuse and the uniform diffuse. The specular term describes mirror-like reflections from the mean plane of the surface:

$$
f_{r, S}\left(\mathbf{v}_{\mathbf{i}}, \mathbf{v}_{\mathbf{r}}\right)=\frac{\left|F\left(\theta_{r}\right)\right|^{2} \exp (-g(\sigma, \lambda)) S\left(\mathbf{v}_{\mathbf{i}}, \mathbf{v}_{\mathbf{r}}\right)}{\cos \left(\theta_{i}\right) d \omega_{i}} \Delta
$$

where $F$ is the Fresnel reflectivity, $S$ is a shadowing function, $\sigma$ refers to the surface roughness, $\lambda$ is the wavelength of the incident light, $\Delta$ is a delta dirac function equal to 1 in the specular cone of reflection, and $d \omega_{i}$ is the incident solid angle. The function of the surface roughness $g(\sigma, \lambda)$ is given by the expression $g(\sigma, \lambda)=\left(((2 \pi \lambda) / \sigma)\left(\cos \left(\theta_{i}\right)+\cos \left(\theta_{r}\right)\right)\right)^{2}$. For a smooth surface $S \rightarrow 1$ and $g \rightarrow 0$, hence the expression of the specular term becomes the specular reflectivity of a specular surface. As for the diffuse directional term, it describes diffraction and interference effects, which spread out the reflected field over the hemisphere, with a possible directional and nonuniform shape of the light intensity distribution:

$$
f_{r, d d}\left(\mathbf{v}_{\mathbf{i}}, \mathbf{v}_{\mathbf{r}}\right)=\frac{\Im(\mathbf{b}, \mathbf{p}) S \tau^{2}}{\cos \left(\theta_{r}\right) \cos \left(\theta_{i}\right) 16 \pi} \sum_{m=1}^{+\infty} \frac{g^{m} e^{-g(\sigma, \lambda)}}{m ! m} e^{\left(-\frac{\mathbf{w}_{\mathbf{v}}{ }^{2} \tau}{4 m}\right)} .
$$

The directional diffuse reflection depends on surface roughness $\sigma$ and on the autocorrelation length $\tau$. The other parameters are the bisecting unit vector $\mathbf{b}$, the incident polarisation state vector $\mathbf{p}$ and the wave vector change $\mathbf{w}_{\mathbf{v}}$. For very smooth surfaces $f_{r, d d}$ decreases to zero and for slightly rough surfaces the maximal values are aligned with the specular direction. As the roughness is increased the maximal values progressively move from off-specular angles to grazing angles for very rough surfaces. The uniform diffuse term is approximated with a Lambertian model and denoted by $f_{r, u d}\left(\mathbf{v}_{\mathbf{i}}, \mathbf{v}_{\mathbf{r}}\right)=a(\lambda)$. An experimental analysis reported in [NDM05] indicates that when polarisation and spectral dependencies are omitted, the He et al. model does not produce noticeably better visual results to the Cook-Torrance model [CT82]. The 


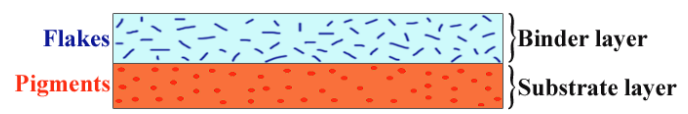

Figure 7: Composition of a two-layer paint used in the Ershov et al. model. Figure adapted by the authors from [EKM01]

model does not suggest a sampling method and does not describe anisotropic materials.

Oren-Nayar [ON94] enhanced the Lambertian model for rough diffuse surfaces, to describe in a more realistic way the behaviour of real-world materials like concrete, sand and cloth, which show increasing brightness as the viewing direction approaches the light source direction, rather than being independent of the viewing direction. A rough diffuse surface is modelled as a collection of long symmetric V-cavities, each of which consists of two microfacets with a Lambertian reflectance; microfacets orientated toward the light source diffusely reflect some light back to the light source (backscatter). The model takes into account masking, shadowing and inter-reflections. A functional approximation of the model, that is easier to compute than the analytical expression, is given by:

$$
f_{r}\left(\mathbf{v}_{\mathbf{i}}, \mathbf{v}_{\mathbf{r}}\right)=\frac{\rho_{d}}{\pi}\left(A+B \max \left(0, \cos \left(\phi_{i}-\phi_{r}\right)\right) \sin (\alpha) \tan (\beta)\right)
$$

where $\alpha=\max \left(\theta_{r}, \theta_{i}\right) ; \beta=\min \left(\theta_{r}, \theta_{i}\right)$; given the surface roughness $\sigma$, the expressions for $A$ and $B$ are:

$A=1-\left[\left(0.5 \cdot \sigma^{2}\right) /\left(\sigma^{2}+0.33\right)\right] ; \quad B=\left(0.45 \cdot \sigma^{2}\right) /\left(\sigma^{2}+0.09\right)$. This model, widely used in computer graphics, obeys the reciprocity principle and reduces to the Lambertian model when $\sigma=0$. The main drawback of the model is that it does not provide specular peaks [DRS07].

The multilayered model by Ershov et al. [EKM01] represents car paint and consists of binder pigment particles, flakes and flake coatings. The model approximates the BRDF of each sub-layer and then merges sub-layers together and it is able to produce realistic appearance for car paints and models their components (binder, pigment particles, flakes). However, due to the complexity of the layered model, the computational time is significantly high. An updated version of the model is simplified to a bi-layered model and presents a substrate layer as a solid paint film where the reflectance is Lambertian and a transparent binder layer with embedded flakes (see Figure 7). Flakes are considered as partially transparent coloured mirrors, with the assumption that the reflectance of flakes does not depend on the incident direction and interreflections between flakes, so their interaction with light is modelled using constant reflectance values [EDKM04]. A large number of parameters are required and not all of them can be directly measured. This model is suitable for interactive design of automotive paints, by solving through optimisation the problem of finding pigment composition of a paint from its bidirectional reflectance distribution function.

To simulate both smooth and rough multi-layered materials, Weidlich and Wilkie [WW07] proposed to combine several microfacet based layers into a single physically plausible BRDF model. Their model assumes that any microfacet is large in relation to the layer thickness, models the absorbtion of part of light when it trav- els inside a transparent material and include a total reflection term, when light propagates at an angle of incidence greater than the critical angle; the simplicity of the model does not allow reproducing effects like iridescence.

Another method aimed to describe the complex reflectance behaviour of a car paint is described in Rump et al. [RMS*08], which represents the reflectance with the first hybrid analytical BRDF and image-based BTF representation; the acquisition setup is described in Section 4.2. The appearance of metallic car paint is separated into the homogeneous BRDF part, which describes the reflection behaviour of the base and the top layer of the paint, and the spatially varying BTF part, which is caused by the aluminium flakes. The homogeneous part is represented by a multi lobe version of the Cook Torrance model [CT82]. In order to account for the characteristics of pearlescent paint, which show view-dependent offspecular colour changes, the model includes a spectral view and light dependent part. The BRDF parameters are derived from the BTF measurements by means of a fitting procedure; the BRDF is calculated for every pixel and subtracted in the RGB space from the captured images. The resulting images contain only flakes data and they are used for a copy and paste synthesis approach.

Kurt et al. [KSKK10] proposed a BRDF model based on the halfway vector representation and Beckmann distribution. The model is physically plausible, can represent anisotropic materials, can accurately fit data and suggests an efficient importance sampling method, based on the strategy proposed by Ward et al. but with a different weighting function, which makes it particularly suitable for Monte Carlo Rendering algorithms. The basic BRDF model they propose is the sum of a pure Lambertian term and a single specular lobe, which can be readily extended to multiple specular lobes representation, to model mixture materials like a car paint:

$$
f_{r}\left(\mathbf{v}_{\mathbf{i}}, \mathbf{v}_{\mathbf{r}}\right)=\frac{k_{d}}{\pi}+\sum_{l=1}^{N} \frac{k_{s l} \Im_{l}\left(\mathbf{v}_{\mathbf{r}}, \mathbf{h}\right) D_{l}(\mathbf{h})}{4\left(\mathbf{v}_{\mathbf{r}} \cdot \mathbf{h}\right)\left(\left(\mathbf{v}_{\mathbf{i}} \cdot \mathbf{n}\right)\left(\mathbf{v}_{\mathbf{r}} \cdot \mathbf{n}\right)\right)^{\alpha_{l}}}
$$

where $N$ is the number of lobes, $k_{d}$ is the diffuse albedo, $k_{s l}$ is the specular reflectivity per-lobe, $\Im_{l}$ is a per-lobe Fresnel term, $D_{l}$ a per-lobe normalised microfacet distribution, $\alpha_{l}$ is a set of parameters which needs to be chosen carefully to enforce energy conservation.

Low et al. [LKYU12] proposed two isotropic models for glossy surfaces, based either on the Rayleigh-Rice light scattering theory (smooth surface BRDF) or on the microfacet theory (microfacet BRDF). Both models make use of a modified version of the ABC model [CTL90, CT91], which was originally formulated to fit the Power Spectral Density of some measured smooth surfaces. The PSD describes the surface statistics in terms of the spacial frequencies $f_{x}$ and $f_{y}$, which depend on the wavelength $\lambda$ of the incident light:

$f_{x}\left(\mathbf{v}_{\mathbf{i}}, \mathbf{v}_{\mathbf{r}}\right)=\left(\sin \theta_{r} \cos \phi_{r}-\sin \theta_{i}\right) / \lambda ; f_{y}\left(\mathbf{v}_{\mathbf{i}}, \mathbf{v}_{\mathbf{r}}\right)=\left(\sin \theta_{r} \sin \phi_{r}\right) / \lambda$.

The ABC model [CTL90,CT91] is able to model the inverse power law shape PSD of polished data, and it is given by:

$$
P S D(f)=A^{\prime} /\left(1+B^{2} f^{2}\right)^{\frac{C+1}{2}}
$$




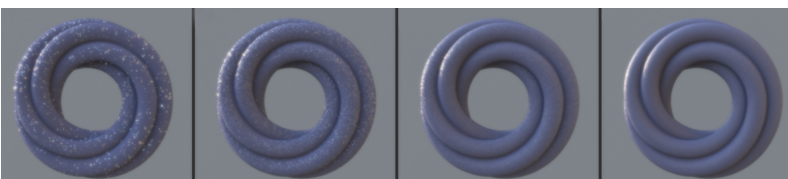

Figure 8: A low number of facets in the [JHY*14] model gives a glittery appearance (left), whereas with an increasing number of microfacets it tends to the classic smooth microfacets appearance (right). (c)2014 Association for Computing Machinery, Inc. Reprinted by permission.

where $\mathrm{A}$ is determined by low-frequency spectral density, $B=2 \pi l_{0}$, $l_{0}$ is the autocorrelation length, $C>0, f=\sqrt{f_{x}^{2}+f_{y}^{2}}, A^{\prime}=\Gamma((c+$ 1) $/ 2) A B /[2 \Gamma(c / 2) \sqrt{\pi}]$ and $\Gamma$ is the gamma function. In [LKYU12] the ABC model is simplified to $S(f)=a /\left(1+b f^{2}\right)^{c}$, where the mapping of the new parameters to the original $\mathrm{ABC}$ is: $a=A^{\prime}$, $b=B^{2}$ and $c=(C+1) / 2$; in practice narrower specular peaks are obtained by increasing $b$, whereas $c$ controls the fall-off rate of wide-angle scattering. The smooth surface BRDF has the following expression:

$$
f_{r}\left(\mathbf{v}_{\mathbf{i}}, \mathbf{v}_{\mathbf{r}}\right)=\left(k_{d} / \pi\right)+O \Im\left(\theta_{d}\right) S\left(\left\|\mathbf{D}_{\mathbf{p}}\right\|\right)
$$

where $k_{d}$ is a scaling factor for the Lambertian term, $O$ is a modified obliquity factor, $\Im\left(\theta_{d}\right)$ is the Fresnel term in Equation 19 with extinction coefficient set to zero and aimed to approximate the reflectivity polarisation factor, which depends on the surface material properties. $\mathbf{D}_{\mathbf{p}}$ is the projected deviation vector, defined as $\mathbf{D}_{\mathbf{p}}=\mathbf{v}_{\mathbf{r}, \mathbf{p}}-\mathbf{r}_{\mathbf{v}, \mathbf{p}}$, where $\mathbf{v}_{\mathbf{r}, \mathbf{p}}$ is the projection of $\mathbf{v}_{\mathbf{r}}$ on the surface tangent plane and $\mathbf{r}_{\mathbf{v}_{\mathbf{i}}}$ is the projection of the mirror direction of $\mathbf{v}_{\mathbf{i}}$ on the surface tangent plane. To deal with unreliable data near grazing angles, the value suggested for the obliquity factor $O$ is 1 instead of the typical definition of $O=\cos \theta_{i} \cos \theta_{r}$. The microfacet model is based on Cook-Torrance [CT82] and makes use of the modified $\mathrm{ABC}$ distribution:

$$
f_{r}\left(\mathbf{v}_{\mathbf{i}}, \mathbf{v}_{\mathbf{r}}\right)=\frac{k_{d}}{\pi}+\frac{\Im\left(\theta_{h}\right) S(\sqrt{1-\mathbf{h} \cdot \mathbf{n}}) G\left(\mathbf{v}_{\mathbf{i}}, \mathbf{v}_{\mathbf{r}}\right)}{\mathbf{v}_{\mathbf{i}} \cdot \mathbf{n} \mathbf{v}_{\mathbf{r}} \cdot \mathbf{n}}
$$

where $\Im$ and $G$ are the same as in Equation 19, $S$ is the modified $\mathrm{ABC}$ distribution and $k_{d}$ is again a scaling factor for the diffuse component; the parameter $a$ of $S$ is used as a scaling factor for the specular term, hence the distribution is not normalised. The model is reciprocal but does not obey energy conservation. Both models provide accurate fits to measured data, with the microfacet model showing lower errors, and accurately represent scattering from glossy surfaces with sharp specular peaks and nonLambertian wide angle scattering. For both models an efficient importance sampling strategy is suggested.

The discrete stochastic model by Jakob et al. [JHY* 14] extends the microfacet theory by replacing the continuous distribution of microfacets in the Cook-Torrance model [CT82] with a discrete one, thus assuming that a surface consists of a high but finite number of scattering particles. This assumption facilitates modelling a controllable, non-smooth spatially varying BRDF appearance of a glittery surface, like mica flakes, ice crystals, metallic car paint and craft glitter for decorations. The notion of multiscale BRDF is introduced, which takes into account finite areas and solid angles rather than single points and directions:

$$
f_{r}\left(A, \mathbf{v}_{\mathbf{i}}, \omega_{r}\right)=\frac{\left(\mathbf{v}_{\mathbf{i}} \cdot \mathbf{h}\right) \Im\left(\mathbf{v}_{\mathbf{i}} \cdot \mathbf{h}\right) D\left(A, \omega_{h}\right), G\left(\mathbf{v}_{\mathbf{i}}, \mathbf{v}_{\mathbf{r}}, \mathbf{n}\right)}{a(A) \sigma\left(\omega_{r}\right)\left(\mathbf{v}_{\mathbf{i}} \cdot \mathbf{n}\right)\left(\mathbf{v}_{\mathbf{r}} \cdot \mathbf{n}\right)}
$$

where $A$ is the area around the point $p$ into account, $a(A)$ its surface area, $\omega_{h}:=\left\{\left(\mathbf{v}_{\mathbf{i}}+\mathbf{v}_{\mathbf{r}} /\left\|\mathbf{v}_{\mathbf{i}}+\mathbf{v}_{\mathbf{r}}\right\|\right), \mathbf{v}_{\mathbf{r}} \in \omega_{r}\right\}$ is the set of microfacet normals that reflect from $\mathbf{v}_{\mathbf{i}}$ into the finite solid angle $\omega_{r}$ around $\mathbf{v}_{\mathbf{r}}$, $\sigma\left(\omega_{r}\right)$ is the area of $\omega_{r}$ on the unit sphere, $\Im$ is the fresnel term, $G$ models shadowing and masking. The discrete multiscale microfacets distribution $D$ is defined as:

$$
D\left(A, \omega_{h}\right)=\frac{1}{N} \sum_{k=1}^{N} 1_{\omega_{h}}\left(\mathbf{v}_{\mathbf{h}}{ }^{k}\right) 1_{A}\left(p^{k}\right)
$$

where $p^{k}$ and $\mathbf{v}_{\mathbf{h}}{ }^{k}$ are the position and normal of the $k^{t h}$ microfacet of a list of $N$ microfacets, $1_{A}$ and $1_{\omega_{h}}$ are the indicator functions of the sets $A$ and $\omega_{h}$ respectively. The indicator functions control the appearance of the surface, since they determine which microfacets in $A$ reflect light into the solid angle $\omega_{r}$ around $\mathbf{v}_{\mathbf{r}}$ : a high number of participating facets gives a smoother appearance than a low number, which gives instead a strongly glittery appearance (see Figure 8). An efficient implementation of the model is discussed, together with an importance sampling strategy for Monte Carlo renderers.

Dupuy et al. [DHI*15] proposed an approach to automatically convert an arbitrary material to a microfacet BRDF. The facet distribution is obtained by solving an eigenvector problem, based solely on backscattering samples and simplifying the Fresnel term to a constant; once an eigenvector with all positive components is found with the power iteration method, its values are linearly interpolated to build a continuous distribution. The Fresnel term is then recovered by calculating for each colour channel the average ratio between the input and an ideal mirror microfacet BRDF with a constant Fresnel term equal to 1 , in the form $f_{r}\left(\mathbf{v}_{\mathbf{i}}, \mathbf{v}_{\mathbf{r}}\right)=$ $D(\mathbf{h}) G\left(\mathbf{v}_{\mathbf{i}}, \mathbf{v}_{\mathbf{r}}\right) /\left(4 \cos \theta_{i} \cos \theta_{r}\right)$. This method can fit an anisotropic BRDF in a few seconds and allows to edit the properties of the roughness distribution, however its accuracy depends on the density of the measurements in the backscattering direction, thus limiting the applicability to more complex anisotropic BRDFs [FHV15].

\subsection{Data-driven models}

A continuous function can be represented by a linear combination of basis functions and a mixture of basis functions can be used for interpolation. In the Fourier basis, the functions are expressed as a sum of sinusoidal and cosinusoidal terms. In the polynomial basis a collection of quadratic polynomials are used with real coefficients.

A possible way to represent BRDFs is to project them onto an orthonormal basis [AMHH08], mapped onto a unit disc and projected on to a hemisphere. Spherical wavelets, spherical harmonics and Zernike polynomials are mathematically and computationally efficient, since they represent the shape of the BRDF as the sum of low and high frequency functions to capture the shape of the BRDF. Wavelets [LF97, CPB03, CBP04] can represent large specular peaks more efficiently than spherical harmonics [WAT92] and Zernike polynomials [Rus98]. The limitation of these methods is 
the significant memory requirement even to obtain simple BRDFs, since a large number of basis functions is generally required.

When a non-linear function is used for BRDF data fitting there are several shortcomings, due the number of parameters which can be large depending on the model and the number of lobes, and to the non-linear estimation process which can be computationally expensive. Ozturk et al. [OKBG08] proposed a representation based on response surface models, defined as a polynomial function of order $p$ in $k$ variables, and expressing a BRDFs as functions of the incoming and outgoing direction and transforming the variables of some non-linear reflectance models (specifically Ward [War92], Lafortune [LFTG97] and Ashikhmin- Shirley [AS00], described in Section 3.1) using Principal Component Analysis, thus obtaining a linear representation. This reciprocal but not energy preserving representation is general enough to model both isotropic and anisotropic materials, diffuse and glossy.

Separable decompositions of a high-dimensional function $f$ can be used to approximate it to arbitrary accuracy, using a sum of products of lower-dimensional functions. Four dimensional BRDFs can be written as a sum of terms each of which is the product of twodimensional functions $g(\cdot, \cdot)$ and $h(\cdot, \cdot)$ :

$$
f_{r}\left(\mathbf{v}_{\mathbf{i}}, \mathbf{v}_{\mathbf{r}}\right)=f_{r}\left(\theta_{i}, \phi_{i}, \theta_{r}, \phi_{r}\right) \approx \sum_{k=1}^{N} g_{h}\left(\theta_{i}, \phi_{i}\right) h_{k}\left(\theta_{r}, \phi_{r}\right) .
$$

This representation directly approximates the fully tabulated BRDF over all directions, implementing manageability of data for use in rendering systems and it is also useful for the purpose of importance sampling. If a good approximation can be found for a small $N$, a separable decomposition is capable of high compression rates, thus resulting in a compact way to store large measured datasets, while maintaining accurate representation. The parameterisation of the lower-dimensional functions can improve the performance of the decomposition and needs to be wisely chosen in order to minimise the number of functions needed for BRDF representations. A common reparameterisation makes use of the angle halfway the incident and exitant directions and the difference angle [Rus98] (see Figure 2):

$$
f_{r}\left(\mathbf{v}_{\mathbf{i}}, \mathbf{v}_{\mathbf{r}}\right) \approx \sum_{k=1}^{n} g_{k}\left(\mathbf{v}_{\mathbf{h}}\right) h_{k}\left(\mathbf{v}_{\mathbf{d}}\right) .
$$

where $\mathbf{v}_{\mathbf{h}}$ and $\mathbf{v}_{\mathbf{d}}$ arise from the re-parameterisation.

A common technique to obtain a separable representation is the Singular Value Decomposition (SVD) [PFTV88]. Given a matrix $M$ its SVD is the factorisation in the form $M=U S W^{T}$, where $S=\operatorname{diag}\left(\sigma_{k}\right)$ is a diagonal matrix of singular values; the columns of $U=\left[\mathbf{u}_{k}\right]$ and $W=\left[\mathbf{w}_{k}\right]$ are orthonormal. The matrix $M$ can be written as:

$$
M=U S W^{T}=\sum_{k=1}^{K} \sigma_{k} \mathbf{u}_{k} \mathbf{w}_{k}^{T}
$$

where $\mathbf{u}_{k} \mathbf{w}^{T}{ }_{k}$ is an outer product. In Fournier [Fou95] the SVD decomposition is used to approximate the Blinn-Phong BRDF using Ward's measured data; the BRDF is approximated with a sum of terms each of which is the product of two functions, one of the incident and one of the outgoing direction.
A technique for separable decomposition of BRDFs based on either Singular Value Decomposition (SVD) (eq. 42) or Normalised Decomposition (ND) (eq. 43) is described by Kautz and McCool [KM99]. In both cases the separable decomposition $f_{s d, r}$ to approximate the BRDF $f_{r}$ has the form:

$$
f_{r}\left(\mathbf{v}_{\mathbf{i}}, \mathbf{v}_{\mathbf{r}}\right)=f_{s d, r}\left(\mathbf{P}_{\mathbf{x}}\left(\mathbf{v}_{\mathbf{i}}, \mathbf{v}_{\mathbf{r}}\right), \mathbf{P}_{\mathbf{y}}\left(\mathbf{v}_{\mathbf{i}}, \mathbf{v}_{\mathbf{r}}\right)\right)
$$

where $P_{x}$ and $P_{y}$ are vector functions. In the following, the parameters of $f_{s d, r}$ are $\mathbf{x}=\mathbf{P}_{\mathbf{x}}\left(\mathbf{v}_{\mathbf{i}}, \mathbf{v}_{\mathbf{r}}\right)$ and $\mathbf{y}=\mathbf{P}_{\mathbf{y}}\left(\mathbf{v}_{\mathbf{i}}, \mathbf{v}_{\mathbf{r}}\right)$. The matrix $M$ of Equation 39 consists of the tabulated and reparameterised BRDF values of $f_{s d, r}(\mathbf{x}, \mathbf{y})$ :

$$
M=\left(\begin{array}{ccc}
f_{s d, r}\left(\mathbf{x}_{1}, \mathbf{y}_{1}\right) & \cdots & f_{s d, r}\left(\mathbf{x}_{1}, \mathbf{y}_{K}\right) \\
\vdots & \ddots & \vdots \\
f_{s d, r}\left(\mathbf{x}_{K}, \mathbf{y}_{1}\right) & \cdots & f_{s d, r}\left(\mathbf{x}_{K}, \mathbf{y}_{K}\right)
\end{array}\right)
$$

The resulting $\mathbf{u}_{k}$ and $\mathbf{w}_{k}$ from the SVD of $M$ can be interpolated in order to obtain the 2D functions $u_{k}(\mathbf{x})$ and $w_{k}(\mathbf{y})$ :

$$
f_{S V D, r}(\mathbf{x}, \mathbf{y}) \approx \sum_{k=1}^{N} \sigma_{k} u_{k}(\mathbf{x}) w_{k}(\mathbf{y})
$$

As for the ND factorisation:

$$
f_{N D, r}(\mathbf{x}, \mathbf{y}) \approx g_{1}(\mathbf{x}, \mathbf{y}) h_{1}(\mathbf{x}, \mathbf{y})
$$

where $g_{1}$ is a constant if $\mathbf{P}_{\mathbf{x}}\left(\mathbf{v}_{\mathbf{i}}, \mathbf{v}_{\mathbf{r}}\right)$ is fixed and scales the profile $h_{1}$. While SVD can produce optimal approximations and minimises the RMS error, it is expensive in terms of time and space resources and can produce negative factors in the expansion; the ND algorithm does not guarantee optimality but requires less memory than the SVD decomposition and it is faster. The lower dimensional functions are stored into texture maps, to allow multiplications being performed by compositing or multitexturing.

McCool and Ahmad presented [MAA01] a decomposition algorithm for both isotropic and anisotropic BRDFs. The algorithm is based on logarithmic homomorphism (eq. 44) and it is general enough to approximate BRDFs with an arbitrary number of positive factors and degree of precision, while satisfying the Helmholtz reciprocity, but limited to point and directional light sources. The authors describe a simple parameterisation (eq. 45) and demonstrate that it is possible to limit the storage cost to just two texture maps, obtaining good compression ratios:

$$
\begin{aligned}
\log \left(f_{r}\left(\mathbf{v}_{\mathbf{i}}, \mathbf{v}_{\mathbf{r}}\right)\right) & \approx \sum_{j=1}^{N} \log \left(p_{j}\left(\pi_{j}\left(\mathbf{v}_{\mathbf{i}}, \mathbf{v}_{\mathbf{r}}\right)\right)\right), \\
f_{r}\left(\mathbf{v}_{\mathbf{i}}, \mathbf{v}_{\mathbf{r}}\right) & \approx p\left(\mathbf{v}_{\mathbf{i}}\right) q(\mathbf{h}) p\left(\mathbf{v}_{\mathbf{r}}\right)
\end{aligned}
$$

where $p(\cdot)$ are two dimensional functions and $\pi_{j}$ are projection functions $\mathbb{R}^{4} \rightarrow \mathbb{R}^{2}$. The logarithmic transformation tends to disregard large peaks in the data and smoothes specular highlights, which may lead to high approximation errors.

Lawrence et al. [LRR04] presented an importance sampling algorithm for arbitrary BRDFs, based on reparameterising the BRDFs using the half-angle or the incident angle, followed by a non-negative matrix factorisation, essential for sampling purposes:

$$
f_{r}\left(\mathbf{v}_{\mathbf{i}}, \mathbf{v}_{\mathbf{r}}\right)\left(\mathbf{v}_{\mathbf{i}} \cdot \mathbf{n}\right) \approx \sum_{j=1}^{J} F_{j}\left(v_{r}\right) \sum_{k=1}^{K} u_{j k}\left(\theta_{p}\right) v_{j k}\left(\phi_{p}\right)
$$


The factored form (Equation 46) allows expressing the BRDF, multiplied by the cosine of the incident angle, as a sum of a small number of terms, each of which is a product of a 2D function $F_{j}$ only dependent on the outgoing direction and two $1 \mathrm{D}$ functions $u_{j k}, v_{j k}$ dependent on the angle chosen for the parameterisation $\mathbf{v}_{\mathbf{p}}=\left(\theta_{p}, \phi_{p}\right)$. The 1D functions are used to interpret the factors as 1D probability distributions. The results are generally accurate and the technique can be used for sampling BTFs and light fields, but does not enforce reciprocity and the representation may present a discontinuity at the pole of the angle selected for the parameterisation.

In later work Lawrence et al. [LBAD*06] presented an algorithm based on linear constraint least squares, capable of compact and accurate SVBRDF representation for rendering. Under the assumption that BRDFs are blended linearly over the surface, the matrix factorisation algorithm provides an editable decomposition and can represent directional and spatial reflectance behaviour of a material. The described Inverse Shade Tree (IST) representation takes as input a measured materials dataset and a user-supplied tree structure and fills in the leaves of the tree. IST proceeds top-down at each stage decomposing the current dataset according to the type of node encountered in the tree. The leaves provide editability since they correspond to pieces that are meaningful to the user. The Alternating Constrained Least Squares algorithm (ACLS) decomposes the SVBRDF into basis BRDFs as 4D functions in tabular form which are then decomposed into 2D functions and further into 1D curves. The 1D curves represent data simply and accurately for isotropic materials, for anisotropic materials the decomposition ends into 2D functions. The advantage of ACLS is the possibility to easily add linear constraints, thus allowing to enforce energy conservation, reciprocity and monotonicity, other than sparsity and nonnegativity, but it requires building a regularly sampled data matrix for factorisation.

Matusik et al. [MPBM03a] presented a set of data-driven reflectance models, based on either linear and non-linear dimensionality reduction. A set of 104 isotropic BRDFs, parameterised using the half-angle [Rus98], are discretised into $90 \times 90 \times 180$ bins which are smoothed by removing outliers. The linear dimensionality reduction used is the Principal Component Analysis (PCA), which allows determination of a set of basis vectors that span the linear subspace on which the BRDFs lie. The RGB colour channels are assembled together and analysed in the log space, in order to reduce the difference between specular and non-specular values. A linear combination of a subset of the principal components is used for the reconstruction and in most cases 30-40 components give good results. As for the non-linear dimensionality reduction, the charting algorithm [Bra02] has been used, since it gives good results even with a small number of samples and at the same time it reduces the noise in the data. The idea behind charting is that data lies on a low dimensional manifold embedded in the sample space and tries to find a kernel-based mixture of linear projections to smoothly map the samples on the coordinate system, while preserving local relationships between the sample points. Since each dimension performs a noise suppression in a different direction the error does not decrease monotonically. However they found that the BRDF data lies in a 10D manifold and a 15D manifold would suffice to synthesise new BRDFs even over long distances. The advan- tages of such data-driven BRDF models is the realistic appearance and meaningful parameterisation.

Matusik et al. performed a wavelet analysis for all of the measured isotropic BRDFs [MPBM03b], in order to find the maximum required frequency to sample any arbitrary BRDF correctly. For each BRDF a non-uniform wavelet transform is applied to determine the highest coefficients able to reconstruct the BRDF itself with high precision, while setting to zero the rest of the coefficients. The union of the sets of non-zero wavelet coefficients (about $69,000)$, which show some degree of coherence among different BRDFs, corresponds to a set of wavelet functions called Common Wavelet Basis (CWB). The CWB allows reconstruction of a BRDF by solving a system of linear equations. In the same work a simple approach to represent a new measured BRDF is presented, using a linear combination of the BRDFs in the dataset. Using this data they construct a over-constrained system of equation in the form $P \times C \approx B$, where $P$ is the matrix of the BRDFs in the dataset, $C$ a vector of coefficients and $B$ the new measured BRDF. A subset $X$ of the rows of $P$ is constructed in such a way the ratio between the highest and lowest eigenvalue of the matrix $X^{T} X$ is small. Experimentally they have found that 800 samples are enough to represent a new BRDF.

Romeiro et al. [RVZ08] describe a method for inferring the reflectance of isotropic materials from images, assuming known curved surface with known natural illumination. To reduce the dimension of the BRDF domain, parameterised using the halfway vector and difference angle [Rus98], thanks to the reciprocity assumption it is possible to apply the projection $\phi_{d} \leftarrow \phi_{d}+\pi$; for isotropic materials it is possible to apply the projection onto the domain $\left(\theta_{h}, \theta_{d}, \phi_{d}\right)$ and in case of bilateral symmetry (i.e. if the reflectance of the material shows little changes when $\mathbf{v}_{\mathbf{r}}$ is reflected about the incident plane) it is possible to apply the projection $\phi_{d} \leftarrow \phi_{d}+\pi / 2$. If a material is bivariate, .i.e. it satisfies a further generalisation of isotropy, bilateral symmetry and reciprocity, the projection onto the domain $\left(\theta_{h}, \theta_{d}\right) \in[0, \pi / 2]$ is allowed. A bivariate representation is often sufficient to capture offspecular reflections, retro-reflection and Fresnel effect. Under these assumptions, the resulting $2 \mathrm{D}$ domain is sampled using the functions $s\left(\theta_{h}, \theta_{d}\right)=2 \theta_{d} / \pi$ and $t\left(\theta_{h}, \theta_{d}\right)=\sqrt{2 \theta_{h} / p i}$, which allow to increase the sampling density near specular reflections; since bivariate BRDFs vary slowly over a significant region of their domain, an optimisation framework with a smoothness constraint is employed to recover the BRDF:

$$
\underset{f_{r} \geq 0}{\operatorname{argmin}}\left\|I-L f_{r}\right\|_{2}^{2}+\alpha\left(\left\|\Lambda_{s}^{-1} D_{s} f_{r}\right\|_{2}^{2}+\left\|\Lambda_{t}^{-1} D_{t} f_{r}\right\|_{2}^{2}\right)
$$

where $\mathcal{S}=\left(s_{i}, t_{i}\right)$ is a uniform grid in the BRDF domain, $D_{s}$ and $D_{t}$ are $|\mathcal{S}| \times|\mathcal{S}|$ derivative matrices, $\alpha$ is a regularisation parameter, $\Lambda_{s}$ and $\Lambda_{t}$ are $|\mathcal{S}| \times|\mathcal{S}|$ matrices that control non-uniform regularisation in the $\left(\theta_{h}, \theta_{d}\right)$ domain and $L$ is a lighting matrix. The term $I$ is related to the rendering equation:

$$
I\left(\mathbf{v}_{\mathbf{r}}, \mathbf{n}\right)=\int_{\Omega} L\left(R_{n}^{-1} \mathbf{v}_{\mathbf{i}}\right) f_{r}\left(s\left(\mathbf{v}_{\mathbf{i}}, R_{n} \mathbf{v}_{\mathbf{r}}\right), t\left(\mathbf{v}_{\mathbf{i}}, R_{n} \mathbf{v}_{\mathbf{r}}\right)\right) \cos \theta_{i} d v_{i}
$$

where $R_{n}$ rotates $\mathbf{n}$ towards the $z$-axis and $\mathbf{v}_{\mathbf{r}}$ towards the $x z$-plane. In order to obtain good results, the environment illumination used to capture the 2D picture must allow sufficient observations of the 
$\operatorname{BRDF}\left(\theta_{h}, \theta_{d}\right)$ domain, in particular in regions corresponding to specular reflections, retro-reflections and grazing angles, which occur respectively at $\left(\theta_{h} \approx 0\right),\left(\theta_{d} \approx 0\right)$ and $\left(\theta_{d} \approx \pi / 2\right)$.

Pacanowski et al. [PSCS*12] employ a subset of the halfway parameterisation [Rus98] to project measured BRDFs on the twodimensional space $\left(\theta_{h}, \theta_{d}\right)$ and approximates the projection by using Rational Functions (RF), since they are able to properly approximate the typical steep changes of specular lobes. A RF $r$ of a finite dimensional vector $\mathbf{x}$ of real variables is defined as:

$$
r_{n, m}(\mathbf{x})=\frac{\sum_{j=0}^{n} p_{j} b_{j}(\mathbf{x})}{\sum_{k=0}^{m} q_{k} b_{k}(\mathbf{x})}
$$

where $p_{j}$ and $q_{k}$ are real numbers and $b_{j}(\mathbf{x}), b_{k}(\mathbf{x})$ are multivariate basic functions, for example multinomials. Given $t+1$ measured values $b_{i}$ located at a vector $\mathbf{x}_{i}$ and contained in the intervals $\left[b_{i}, \overline{b_{i}}\right]$, the data fitting problem can be stated as finding a $\mathrm{RF} r_{n, m}(\mathbf{x})$ with the smallest possible $n+m$, to interpolate the $t+1$ intervals with the additional constraints of non-negativity, monotonicity and symmetry: $\forall i=0, \ldots, t \quad b_{i} \leq r_{n, m}\left(\mathbf{x}_{i}\right) \leq \overline{b_{i}}$. The widths of the interpolation intervals are chosen in such a way that the renderings are visually satisfactory, while keeping the number of coefficients reasonably low; the solution is found by solving a quadratic programming problem. Isotropic BRDF data are approximated with a single RF, called Rational BRDF:

$$
f_{r, s}\left(\mathbf{v}_{\mathbf{i}}, \mathbf{v}_{\mathbf{r}}\right) \approx f_{\theta_{h}, \theta_{d}} \approx r_{n, m}\left(\theta_{h}, \theta_{d}\right) .
$$

The anisotropic model is based on the observation that for some anisotropic materials like brushed metals, the variation of the reflected intensity, when the surface is rotated around the normal $\mathbf{n}$, consists of a scaling factor applied to an average isotropic lobe:

$$
f_{r, s}\left(\mathbf{v}_{\mathbf{i}}, \mathbf{v}_{\mathbf{r}}\right) \approx r_{n^{\prime}, m^{\prime}}^{a}\left(\phi_{h}\right) r_{n, m}^{i}\left(\theta_{h}, \theta_{d}\right)
$$

where $r_{n, m}^{i}\left(\theta_{h}, \theta_{d}\right)$ is a isotropic Rational BRDF and $r_{n^{\prime}, m^{\prime}}^{a}\left(\phi_{h}\right)$ is a scaling factor to model anisotropic variations. The same approximation process applied to BRDFs can be applied to the inverse Cumulative Distribution Function (CDF) and used for importance sampling; the use of RF for BRDFs and CDFs allows to obtain a very small memory footprint.

Bilgili et al. [BÖK11] proposed to represent four-dimensional measured BRDFs data as a function of tensor products, factorised using Tucker decomposition [Tuc66], a generalisation of higher order principal component analysis. Tensors are a generalisation of scalars and vectors to higher orders and their rank is defined by the number of directions, e.g. a scalar is a zero-order tensor and a vector a first-order tensor; the Tucker factorization decomposes a tensor into a set of matrices and one small core tensor. The logarithmic transformation of a $4 D \mathrm{BRDF}$ data matrix $B=b_{i j k l}$, based on the halfway vector representation, can be roughly approximated by setting all the Tucker parameters to 1 :

$$
\log \left(b_{i j k l}\right) \approx g f_{1}\left(\theta_{h i}\right) f_{2}\left(\phi_{h j}\right) f_{3}\left(\theta_{r k}\right) f_{4}\left(\phi_{r l}\right)
$$

where $i=1, \ldots, N_{\theta_{h}}, j=1, \ldots, N_{\phi_{h}}, k=1, \ldots, N_{\theta_{r}}, l=1, \ldots, N_{\phi_{r}}$ and $N_{\theta_{h}}, N_{\phi_{h}}, N_{\theta_{r}}, N_{\phi_{r}}$ are the sampling resolution of the BRDF data, $g$ is the zero-order core tensor, $f_{1}\left(\theta_{h i}\right), f_{2}\left(\phi_{h j}\right), f_{3}\left(\theta_{r k}\right), f_{4}\left(\phi_{r l}\right)$ are univariate tensor functions respectively evaluated at $\theta_{h i}, \phi_{h j}, \theta_{r k}$ and $\phi_{r l}$; the logarithmic transformation eliminates the problem of estimated negative BRDF values. The error matrix $\mathbf{e}_{1}$ of this approximation can be written as $\mathbf{B}_{0}=\mathbf{B}_{0}^{\prime}+\mathbf{e}_{1}$, where $\mathbf{B}_{0}=\log \left(b_{i j k l}\right)$ and $\mathbf{B}_{0}^{\prime}$ is the approximation. The approximation is improved by applying recursively the decomposition on the error terms, until a satisfactory level of accuracy is obtained; assuming that $S$ is the total number of iterations, the expression of $\mathbf{B}_{0}$ becomes:

$$
\mathbf{B}_{0} \approx \mathbf{B}_{0}^{\prime}+\mathbf{e}_{1}^{\prime}+\mathbf{e}_{2}^{\prime}+\ldots+\mathbf{e}_{S-1}^{\prime}
$$

where $\mathbf{e}_{1}=\mathbf{e}_{1}^{\prime}+\mathbf{e}_{2}$ and $\mathbf{e}_{1}^{\prime}$ is the Tucker approximation of $\mathbf{e}_{1}, \mathbf{e}_{2}$ is the error term of the second and so on. This non-negative representation allows good compression ratios while being able to represent Fresnel effects and off-specularities, but does not satisfy reciprocity and energy conservation. As for the importance sampling, to limit the sampled region for isotropic materials it has been shown that most of the total variation is due to two components which corresponds to univariate functions of $\theta_{h}$ and $\theta_{r}$; a similar property is observed for the anisotropic material, where the main components are univariate functions of $\theta_{h}$ and $\phi_{h}$.

Tensor representation has been previously used for interactive modification of the material properties and relighting by Sun $e t$ al. [SZC ${ }^{*}$ 07]. Based on the observation that high-frequency specular lobes generally require a large number of basis terms for reconstruction, thus precluding interactive performance, the BRDFs are separated into a specular lobe $f_{r, s}$ and the remainder $f_{r, r m}$. The specular lobe $f_{r, s}$ is modeled as a sum of 4 Gaussians, with different neighborhood support $\left(0^{\circ}\right.$ for perfect mirror reflection, $7^{\circ}, 14^{\circ}$ and $21^{\circ}$ for broader Gaussians). By removing $f_{r, s}$ the BRDF is left with mainly low frequency terms, that can be modeled with a small basis by tensor approximation.

More recently tensor representation has been used in [WKB12, WKB14] to represent anisotropic materials with no assumption on the reflectance and scattering behaviour, particularly useful in presence of unusual scattering properties. The measured data is fitted to a series of radial basis functions in order to derive a continuous representation from the sparse input 4-D measurements. The incident and reflected hemispheres are projected onto disks and mapped over the unit square; the four dimensions given by the two squares define a rank-4 tensor, subdivided into a tensor tree for fast Monte Carlo sample generation. The tensor tree representation adaptively subdivides sharp peaks of the BRDF in different regions of the distribution, with an additional averaging step between incident and reflected direction to account for Helmoltz reciprocity.

\section{Reflectance acquisition setups}

Measuring or calculating how a surface interacts with light is a time consuming and expensive procedure, which generates a vast amount of data, but it is important for realistic appearance of a material model. BRDF measurements are not only used in Computer Graphics to reproduce material reflectance, but also in many other fields such as Computer Vision (e.g. in object recognition applications), Aerospace (e.g. for optimal definition of satellite mirrors reflectance and scattering properties), Optical Engineering, RemoteSensing (e.g. land cover classification, correction of view and illumination angle effects, cloud detection and atmospheric correc- 


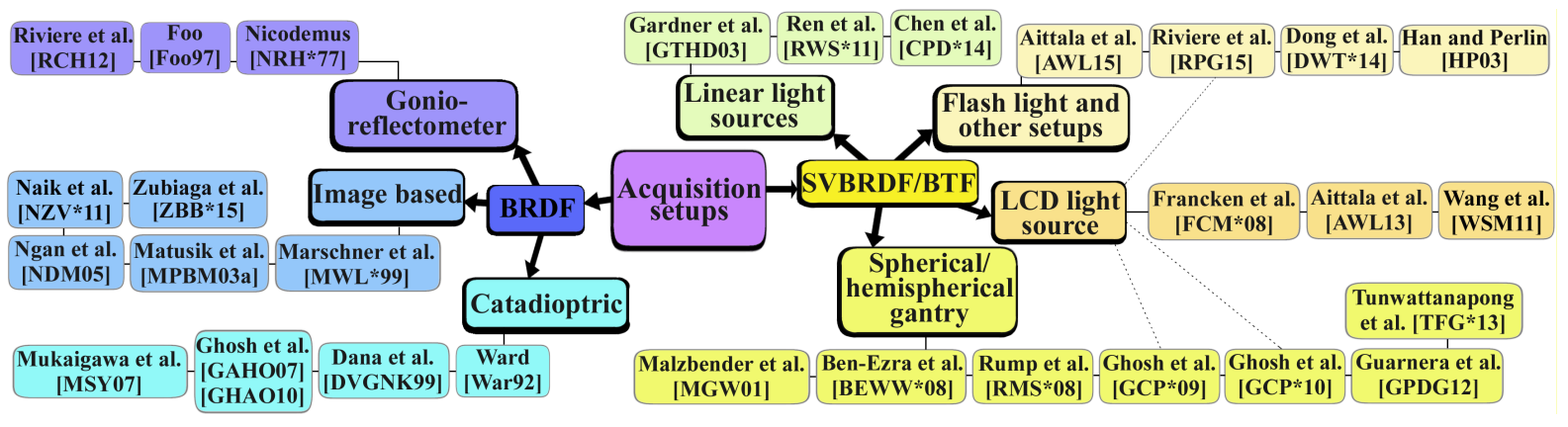

Figure 9: Taxonomy of the BRDF/SVBRDF acquisition setups described in Section 4.

tion), Medical applications (e.g. diagnostics), Art (e.g. 3D printing), Applied Spectroscopy (e.g. physical condition of a surface).

The setup of a typical measurement device includes a light source to uniformly illuminate a large area of a surface and a detector to measure a small area within the illuminated region [ASMS01]. Various systems with different degrees of accuracy and costs have been constructed to measure reflectance functions, ranging from gonioreflectometers to image based measurement systems; low cost setups have also been investigated [HP03, FHV15, RLCP]. By dropping the assumption that a material is homogeneous and opaque, many techniques for BRDF measurement can be adapted for more complex reflectance functions (SVBRDFs, BTFs, BSSRDFs). Under certain assumptions, also setups used to acquire objects geometry through the classical photometric stereo technique [Woo80], where the point of view is kept constant between successive images while the direction of incident illumination varies, have been successfully used to recover BRDF and SVBRDF of non-lambertian surfaces [Geo03, GCHS05, HS05, CGS06,ZREB06, AZK08, HLHZ08]. Some of these techniques are limited to materials with a single specular lobe [GCHS05] due to the use of optimisation algorithms to recover the parameters for the Ward isotropic BRDF [War92] or require the acquisition of reference objects of known shape and with similar materials as the target [HS05]; to reduce the number of input pictures it has been assumed bivariate BRDFs [AZK08] or spatial coherence of reflectance, trading spatial for angular resolution [ZREB06]. The taxonomy of the acquisition setups, detailed in the following sections, is reported in Figure 9.

To assess the quality of an acquisition setup it is important to derive a standardised error between the measured appearance model and the original object [GLS04]. The distance metric $\Delta E$ is particularly suitable to measure colour differences; it is defined in the CIE XYZ colour space [WS82], a perceptually uniform space which describes the chromatic response of a standard human observer to the lighting stimulus, accounting for the incident spectral power distribution. For digital image sensors, at the heart of image based systems, the most common colour space is $s R G B$, which due to the characteristics of the Human Visual System is often prone to inaccuracies [Fai05]. Acquired RGB values could be translated into the $C I E X Y Z$ and post-processed for white balancing [WEV02], although metamerism (i.e. spectra that appear identical to a human observer under a certain light) would still represent a source of er- rors. A more robust solution would make use of a carefully characterised acquisition device (e.g. a DSLR camera), to obtain either a relative [KK08] or an absolute colorimetric estimation of the scene in $c d / m^{2}$ [GBS14].

A number of commercial BRDF measurement devices and systems are currently available, including gonio-photometers and reflectometers (pgII gonio-photometer [AB14], SOC-200 $B D R\left(^{*}\right)$ [Sur16], $\operatorname{Reflet}\left(^{*}\right)$ [Lig16], gonio'2pi $\left(^{*}\right)$ [Ops16], CASI [Sch16]), hemispherical measurement domes (IS-SA [Rad16]) and devices aimed for specific categories of objects and surfaces, for instance displays (DMS 201/505/803(**) [Kon16]) and car paint (the handheld MA98 spectrophotometer [ X-16]); at the time of writing, for some of the mentioned devices several measurement services are also provided by the producing companies (indicated by $\left({ }^{*}\right)$ ), or rental programs are offered $\left({ }^{* *}\right)$.

Numerical simulation [CMS87, WAT92, HK93, APS00, DWMG15] represents, for some complex materials, a possible alternative to a measurement device. The material appearance is described by the result of the simulation of the light interaction with the surface (and sub-surface) structure. Given a geometry that can be ray-traced, Westin et al. in their seminal work [WAT92] describe a method to simulate scattering hierarchically, by using the result of the simulation at a scale to generate the BRDF for a larger scale.

\subsection{Gonioreflectometers}

The gonioreflectometer measures the spectral reflectance of surfaces, it covers specular and diffuse reflectance depending on the settings of the device. The construction of the device was described by Nicodemus and used in the experimental development of the reflection models by Torrance and Sparrow [TS67], Blinn [Bli77], He et al. [HTSG91] and many others. A detailed setup is described by Hsia and Richmond [HR76]. It consists of a light source (a laser beam), a sample material placed on the sample holder mounted on a turntable which rotates around the vertical axis and a detector which captures data about reflected light from the sample. The aluminium sample holder, painted with matte black paint, is mounted on the arm attached to the turntable and placed in front of the sample detector. Two averaging spheres, with the inside part coated with barium-sulfate, are used to measure the incident light. 


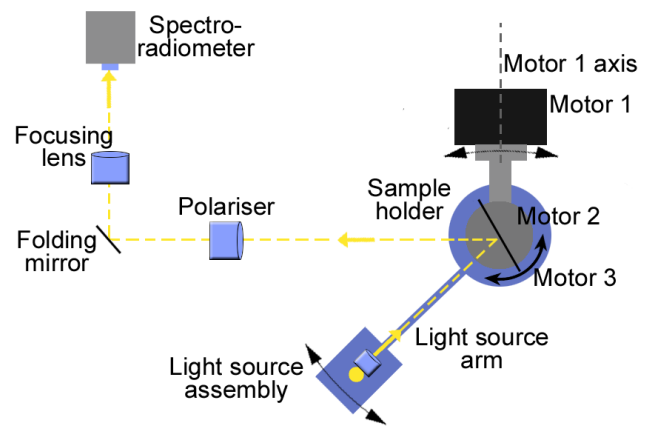

Figure 10: Schematic representation of the three-axis gonioreflectometer described in [Foo97, LFTW06].

Foo [Foo97] designed a three axis automated gonioreflectometer with two degrees of freedom. The measuring system consists of a light source moving around a sample, a stationary detector and a folding mirror. The system can measure the reflection at high grazing angles (up to 86 degrees) and allows high dynamic range measurements, making it considerably precise. Unfortunately, this setup can only measure isotropic BRDFs. A similar setup is described by Li et al. [LFTW06] and reported in Figure 10.

Riviere et al. [RCH12] used an in-plane multispectral polarised reflectometer. The measurement setup consists of a lighting system with three linearly polarised laser sources; the polarised detection system is based on the Fresnel equation to identify polariser's axes. It allows sampling at zero lighting angles and it is fully calibrated for polarised and multispectral in-plane BRDF measurements. Polarised measurements are used to distinguish the different scattering processes in BRDF directional components. This measurement system is suggested for analysis of physical measurements of the optical surface and for laser-imaging applications. It allows users to retrieve BRDF data which prove to be numerically stable; an inversion algorithm is required for high angular measurements of BRDFs [RCH12].

\subsection{Image based measurement}

Image-based BRDF measurement makes use of photographs of an object and requires only general-purpose equipment, thus lowering the cost of the process. The data can be measured quickly and completely through a series of photographs taken of a surface. These photographs capture light reflected from various surface orientations. However, to measure the wavelength spectrum of the BRDF requires more time per measurement [MD98].

Marschner et al. [MWL $\left.{ }^{*} 99\right]$ presents a rapid, complete and accurate isotropic BRDF measurement setup for a broad range of homogeneous materials, including human skin. It can achieve high resolution and accuracy over a large range of illumination and reflection directions. This setup consists of a hand-held digital camera, equipped with a standard CCD sensor with RGB colour filter array, and an industrial electronic flash light source, which suffice to measure surfaces with simple shapes, e.g. spherical and cylindrical which can be defined analytically; for more complex irregular shapes a 3D scanner is required in addition. The camera, charac-
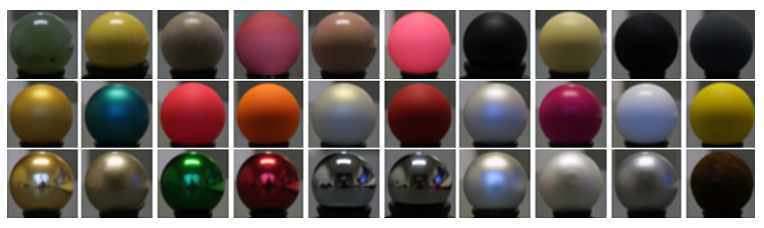

Figure 11: Subset of 30 materials, out of the 100 in the MERL-MIT BRDF database by Matusik et al. [MPBM03a]. Copyright (C)2006 Mitsubishi Electric Research Laboratories All Rights Reserved.

terised in terms of Optoelectronic Conversion Function (OECF) in order to know the radiance reflected to the camera and the irradiance due to the source, moves from near the light source, to measure near retro-reflections, to opposite the light source, in order to measure grazing-angle reflection. Some additional photographs are taken to measure the location and intensity of the light source, the camera pose and the sample pose. About 30 images from different positions are required to cover the three-dimensional BRDF domain. Each pixel in the images is used to derive one sample in the domain of the BRDF, thanks to the estimated relationship between the geometry of the sample and the position of the camera, light source and sample, through bundle adjustment. A typical measurement session takes up to half an hour.

A more recent development by Matusik et al. [MPBM03a] similar to Marschner et al. [MWL ${ }^{*} 99$ ], has been used to measure 100 isotropic materials. The database, which is to date the largest and most reliable BRDF database in the field [AP07], is partly shown in Figure 11. Matusik's data-driven method is described in Section 3.3.

Ngan et al. [NDM05] presented an anisotropic BRDF acquisition setup for flat and flexible samples, depicted in Figure 12. To deal with the anisotropy, strips of the material at different orientations obtained from flat samples are wrapped around a cylinder, which can be tilted by means of a precision motor in order to account for the missing degree of freedom with respect to a sphere. A light source rotates around the cylinder while the target is captured by a fixed camera, enabling the capture of the full $4 \mathrm{~d}$ BRDF. For each light and target position a set of 8 pictures with different exposures is taken, to form an HDR image. The sampling density of the light and the cylinder tilting can be adjusted to increase the resolution of the measured BRDF, whereas the main limitation in the resolution is due to the limited number of material strips which can be wrapped around the cylinder.

The reflectance acquisition setup proposed by Naik et al. [NZV $\left.{ }^{*} 11\right]$ exploits space-time images captured by a time-offlight camera. Two different setups are described, both based on indirect viewing with 3-bounce scattering and making use of two known Lambertian materials, respectively the source $\mathrm{S}$ and the receiver $\mathrm{R}$, while $\mathrm{P}$ is the patch to measure. In the first setup, the laser illuminates $\mathrm{S}$, and the camera views $\mathrm{R}$, thus measuring $\mathrm{P}$ indirectly (Figure 13(a)). As for the second configuration, it is based on an around the corner viewing in which $\mathrm{P}$ is not directly visible to the camera, whereas $S$ and $R$ are the same surface (Figure 13(b)). The light is multiplexed along different transport paths and some of them might have the same length, hence the light can arrive along 


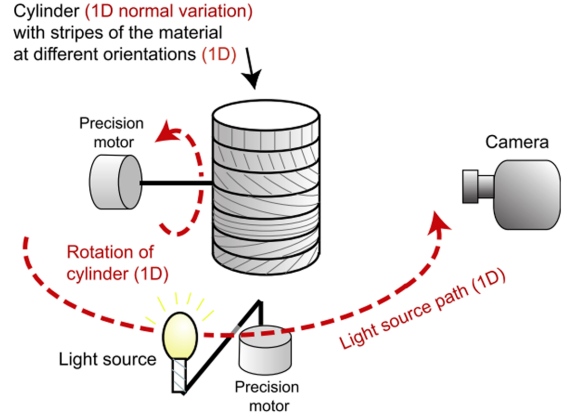

Figure 12: BRDF acquisition setup by Ngan et al. [NDM05]. Copyright (C) 2005 by John Wiley Sons, Inc. Reprinted by permission of John Wiley \& Sons, Inc.

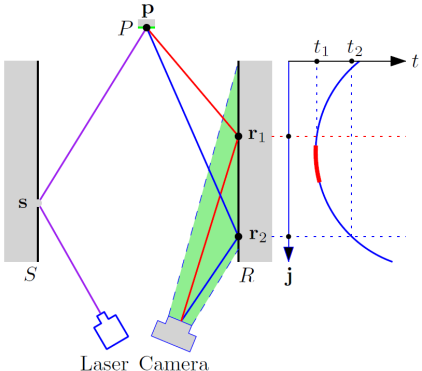

(a) Canonical setup

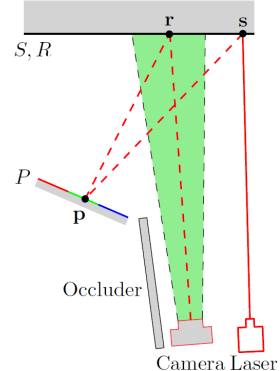

(b) Around-the-corner setup
Figure 13: The two acquisition setups described by Naik et al. [NZV*11]. To measure the patch $P$, the laser illuminates $S$, and the camera images $R$. The shape of the specular lobe of the BRDF of $P$ is recover by analysing the streak image, on the right side of (a). Red indicates regions with higher brightness than the blue part. (C)2011 Association for Computing Machinery, Inc. Reprinted by permission.

multiple paths at the same point at the same time. For this reason the measurements of the material need to be decoded, by solving a sparse underdetermined system; the system is solved by recovering the parameters the Ashikhmin-Premoze model [AP07] (see Section 3.1), using the halfway vector parameterisation. When the multiplexing does not cause ambiguities, in order to measure the parameters of a material it is enough to analyse the streak images to find the specular peak. This setup enables to take many BRDF measurements simultaneously, but it requires an ultra-fast camera; moreover it suffers from a low signal to noise ratio due to the multiple bounces, the size of patches and the maximum sharpness of the reflectance function are limited by the hardware and the range of measurable incoming and outgoing directions is limited by the geometry of the setup.

Whereas the approaches described in this section are aimed to recover BRDF properties from image properties, a recent work by Zubiaga et al. [ZBB*15] aimed to understand how the properties of BRDFs influence the rendered picture, by working locally in Fourier space and analysing how BRDF moments up to order 2 in- duce colouring, warping and blurring of reflected radiance on a subset of 40 unimodal materials in the MERL database [MPBM03a]. The 2D slices of the selected BRDFs are pre-processed with a heuristic method for diffuse and specular separation, and parameterised using a view-centred angular parameterisation with poles orthogonal to the view direction, to minimise distortions around the scattering plane.

\subsection{Catadioptric measurement setups}

Catadioptric optical systems makes use of both reflected and refracted light, in order to reduce aberrations. The resulting imaging setups are generally efficient image based BRDF acquisition devices, usually without any moving parts.

The imaging gonioreflectometer described by Ward [War92] measures anisotropic surfaces by repeating measurement process under various orientations. It captures the entire hemisphere of reflected and refracted directions at the same time. Ward used a fisheye lens and half-silvered hemisphere. This device cannot measure sharp specular peaks nor take measurements at high grazing angles.

Dana et al.'s [DVGNK99] measuring device consists of a robot arm that holds and rotates a sample, a halogen bulb with a Fresnel lens and a video camera. The light is fixed, and the camera is moved to record measurements from seven different locations. The seven location points of the measurements correspond to sample viewing points and illumination direction. For measurement of the anisotropic material the sample is rotated about the z-axis and this procedure is repeated [DVGNK99]. This system was designed for use in computer graphics, and like Ward, includes reflection and refraction capture, however there are issues with noise within measurements and scale as the surface patches are too large to measure fine scale texture variations.

Mukaigawa et al. [MSY07] built a measurement system for anisotropic BRDFs which uses a projector as the light source, placed at the focal point of an ellipsoidal mirror, a camera and a beam splitter, since the camera and the projector cannot be located at the same position. The number of acquired images depends on the sampling of the lighting direction and viewing direction, which needs to be estimated based on the accuracy required. The acquired data are then fitted to the Ward anisotropic reflection model.

Ghosh et al. [GAHO07, GHAO10] describe the setup of a measurement device that does not involve any moving parts and consists of a camera focusing on a zone of reflected directions, a light source with a beam splitter, a mirrored dome and mirrored parabola. The focus of the illumination beam is on the mirrored components that the beam reflects back to its origin. An example of the acquisition is shown in Figure 14. This setup allows BRDF measurement over a continuous region with a specially designed orthonormal zonal basis function illumination, which results in a very rapid BRDF acquisition and in a better signal to noise ratio compared to point-sampling the incident directions [MSY07]. The measurements are then projected into a spherical harmonics basis or fitted to an analytical reflection model. 

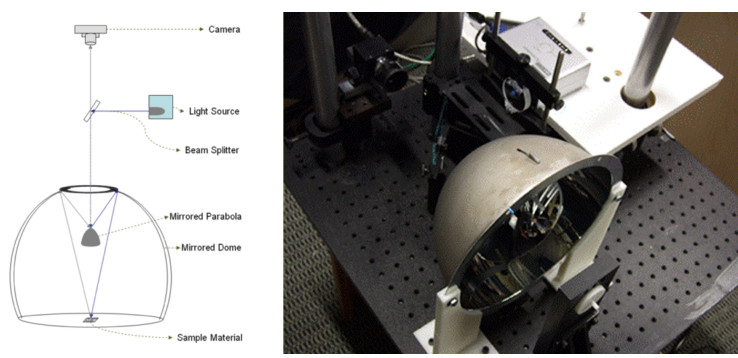

Figure 14: Left: physical reflectance acquisition setup by Ghosh et al. [GAHO07, GHAO10]. On the right a photograph of the actual setup. (C)[2007] IEEE. Reprinted, with permission, from [GHAO10].

\subsection{Spherical and Hemispherical Gantry}

Malzbender et al. [MGW01] built a hemispherical device with 50 strobe light source. The camera is placed in the apex of the device, and it is used to acquire pictures of almost flat samples, placed on the floor and illuminated by a single light source at a time. The acquired data are represented by Polynomial Texture Maps (PTM), in which for each fitted texel the coefficients of the following polynomial are fitted to the data and stored as a map:

$$
\begin{aligned}
& L\left(u, v ; l_{u}, l_{v}\right)=a_{0}(u, v) l^{2}{ }_{u}+a_{1}(u, v) l^{2}{ }_{v}+ \\
& +a_{2}(u, v) l_{u} l_{v}+a_{3}(u, v) l_{u}+a_{4}(u, v) l_{v}+a_{5}(u, v)
\end{aligned}
$$

where $L$ is the surface luminance at $(u, v)$, the local coordinates of the texture and $\left(l_{u}, l_{v}\right)$ are the projection of the normalised light vector at that coordinate. PTMs facilitate good quality rendering, in particular for diffuse samples.

A hemispherical device for anisotropic BRDF measurement was presented by Ben-Ezra et al. [BEWW*08], in which it is demonstrated that with an accurate radiometric and geometric calibration LEDs can be used as light sources and as detectors, without needing any moving parts nor cameras; this setup allows fast acquisition times. In their implementation 84 LEDs pointing toward the centre of the hemisphere are used. During the acquisition, each LED is switched on, in turn acting as an emitter, while all others measure the reflected light from the sample, see Figure 15. The SNR of the measurements can be increased by multiplexed illumination and the use of different colours for the LEDs allows capture of multispectral data. Since a LED cannot be used at the same time as an emitter and detector this setup cannot be used to measure retro-reflection and offers a lower resolution compared to camera-based setups.

The measurement device presented by Rump et al. [RMS*08] consists of a hemispherical gantry with 151 cameras uniformly distributed; the cameras flashes are used as light sources and for each flash all the cameras take a picture of the subject, giving a total of $151 \times 151=22,801$ pictures, which can be increased by taking HDR sequences. The gantry is capable of supporting projectors in order to project structured light on the subject.

Ghosh et al. [GCP*09] proposed three different setups to estimate spatially varying BRDFs for both isotropic and anisotropic

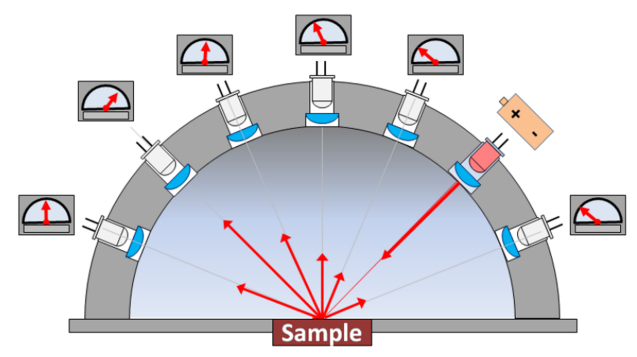

Figure 15: An LED-only BRDF Measurement Device by Ben-Ezra et al. (C)[2008] IEEE. Reprinted, with permission, from [BEWW* 08 ]

materials, using up to 9 polarised second order spherical gradient illumination patterns. For specular reflections, specular albedo, reflection vector and specular roughness can be directly estimated from the $0^{\text {th }}, 1^{\text {st }}\left[\mathrm{MHP}^{*} 07\right]$ and $2^{\text {nd }}$ order $\left[\mathrm{GCP}^{*} 09\right]$ statistics respectively. The first setup, suitable for roughly specular objects of any shape, is based on a LED sphere with 150 controllable lights linearly polarised, with the subject placed at the centre of the sphere. The second setup is suitable for flat objects and uses as the light source a LCD monitor, placed very close to the subject, which clearly offers a smaller coverage of incident direction but with a higher resolution than the LED sphere. The third setup makes use of a roughly specular hemisphere which reflects the light emitted by a projector on the subject placed at the centre of the hemisphere, thus allowing a dense sampling; the camera observes the subject from the apex of the hemisphere.

The analysis of the Stokes reflectance field of circularly polarised spherical illumination has been exploited by Ghosh et al. [GCP*10] to estimate the specular and diffuse albedo, index of refraction and specular roughness for isotropic SVBRDFs, assuming known surface orientation. Three different setups are used to demonstrate the technique, similar to the ones described in $\left[\mathrm{GCP}^{*} 09\right]$ but with the light sources covered with right circular polarisers. Four pictures of the subject are required to measure the Stokes field, three of them with differently oriented linear polarisers in front of the camera and one with a circular polariser.

The same framework based on the analysis of the Stokes reflectance field has been further exploited by Guarnera et al. [GPDG12] and it is extended to cover also unpolarised illumination, to obtain a per-pixel estimate of the surface normal from the same input data as in [GCP*10]. The proposed setup makes use of a LED sphere with 346 controllable lights unpolarised/circularly polarised; the surface normals estimation is demonstrated also with uncontrolled outdoors measurement under overcast and hence unpolarised sky, by capturing a reference dielectric sphere in the same environment.

Tunwattanapong et al. [TFG*13] proposed a spinning spherical reflectance acquisition apparatus as shown in Figure 16. A $1 \mathrm{~m}$ semi-circular arc with 105 LED focused toward the centre rotates about the vertical axis at $1 \mathrm{rpm}$, sweeping out continuous spherical harmonic illumination conditions. They demonstrated that 44 pictures are enough to estimate anisotropic SVBRDFs and the 3D 


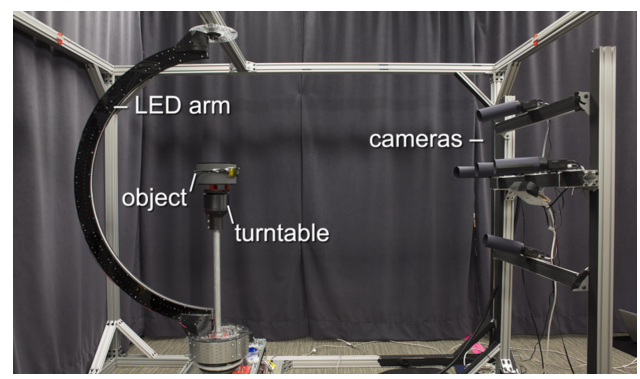

Figure 16: Capture setup by Tunwattanapong et al. [TFG* 13] (C)2013 Association for Computing Machinery, Inc. Reprinted by permission.

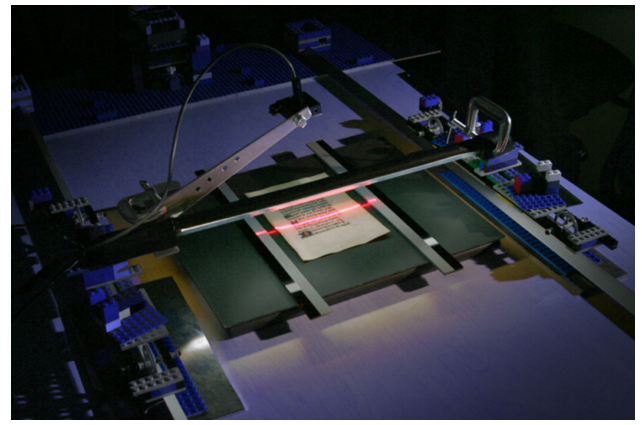

Figure 17: Acquisition setup by Gardner et al. [GTHD03] (c)2003 Association for Computing Machinery, Inc. Reprinted by permission.

geometry of very specular or diffuse objects. This technique further generalises the approach by Ghosh et al. [GCP*09], since it can be applied to higher-order spherical harmonic illumination (up to $5^{\text {th }}$ order), which allows obtaining diffuse/specular separation without relying on polarisation.

Gardner et al. [GTHD03] built a low cost linear light source apparatus to capture flat samples (Figure 17) making use of a fixed camera for imaging and a structured light diode. The light source is a $50 \mathrm{~cm}$ long neon tube, which is translated horizontally over the surface of the subject and moved in sync with the camera acquisitions. The reflectance model used to fit the measured data is the isotropic model by Ward [War92], given the camera and light source positions at each frame. The laser projects a laser stripe, which is deformed by surface variations and used in order to recover the geometry, together with two scans of the light source, in a diagonal direction. A cabin light box, with two diffused cathode tubes are used as a sample holder and to project a even diffuse white light on the surface and allows measurement of the transmitted and reflected light. Overall, the system allows recovery of the diffuse and specular colours, specular roughness, surface normals and per pixel translucency for isotropic samples.

In Ren et al. [RWS*11] a hand-held linear light source device, together with a BRDF chart is employed to obtain spatially varying isotropic BRDFs from a video taken with a mobile phone in LDR. The BRDF chart consists of 24 square flat tiles, with known

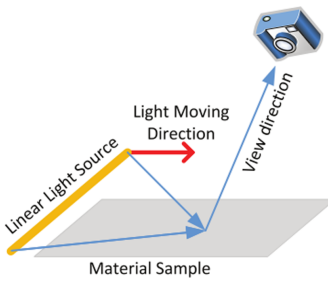

(a) Desktop scanner setup

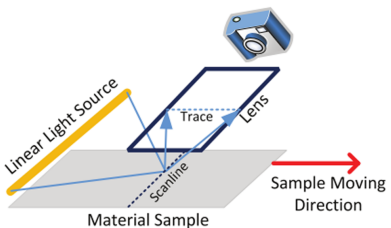

(b) Handheld scanner setup
Figure 18: Generalised linear light source reflectometer by Chen et al. [CDP* 14] (c)2014 Association for Computing Machinery, Inc. Reprinted by permission.

BRDFs. The tiles are made of specular materials, except one which is a diffuse standard for camera calibration (exposure and white balance). The light source is a $40 \mathrm{~cm}$ florescent tube, slowly moved by hand over the surface and the chart, which needs to be placed alongside. This approach requires solving a number of issues, since the camera and the light source need to be placed close to the sample and the light is moved manually. Consequently, the camera and light position are unknown, as well as the SVBRDF of the sample. Saturated values from LDR acquisition are repaired using the values in the neighbourhood and the reflectance responses are normalised and hence aligned by a dynamic time warping algorithm. Aligned samples are then used for BRDF reconstruction.

Chen et al. [CDP*14] present a similar setup to Gardner et al. [GTHD03], scanning a linear light source over a flat sample (Figure 18) but with the significant advantage of capturing anisotropic surface reflectance. The basic assumption is that a microfacet model can be used to model the anisotropic surface reflectance. To observe the specular reflection they modulate the illumination along the light source, by means of a transparent mask. They propose two different setups which differ in form factor and employ the same 35cm CCFL lamp and DSLR camera. The desktop form factor scanner scans a linear light source over the sample, observing the SVBRDF by means of the camera; as for the hand-held form factor scanner, the sample moves with respect to the camera and the linear light source, which instead have a fixed relative position. Finally a cylindrical lens is employed to capture in a single picture a scanline of the sample. One constant lighting pattern, together with two phase shifted sinusoidal patterns suffices to reconstruct the surface reflectance.

\subsection{LCD Light Source}

Francken et al. [FCM $\left.{ }^{*} 08\right]$ make use of commodity hardware such as a LCD display and a SLR camera to recover detailed normal maps of specular objects, based on the observation that the normal of a specular pixel is the halfway vector between the light direction and the view direction. To identify the light direction among $n$ different light sources they make use of a gray code lighting patterns, by taking $O\left(\log _{2} n\right)$ pictures. The accuracy of the estimated normal map depends on the number of sampled light sources.

In Aittala et al. [AWL13] a low cost capture setup for SVBRDFs is presented, as shown in Figure 19 with a similar setup as Francken 


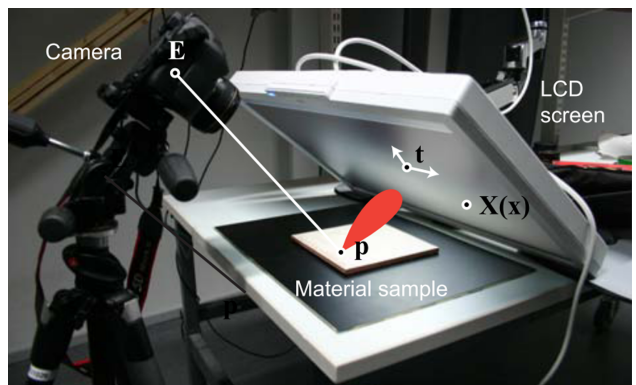

Figure 19: SVBRDF capture in the frequency domain by Aittala et al. [AWL13] (C)2013 Association for Computing Machinery, Inc. Reprinted by permission.

et al. Their work relies on the design of the image formation model and uses a Fourier basis for the measurements. Isotropic BRDFs are reconstructed through Bayesian inference, since the model is analytically integrable.

The capture set up by Wang et al. [WSM11], consists of a vision camera and a regular LCD, used as an area light source (see Figure 20(a)). It allows rapid measurement of a stationary, isotropic, glossy and bumpy surface, describing its appearance with a duallevel model, which consists of the specular and diffuse relative albedos, two surface roughness parameters and a 1D power spectrum over frequencies for visible surface bumps. Two images are required for calibration, since the LCD radiance is dependent on the viewing angle. To establish the pose of the surface with respect to the camera a target is placed on the surface. At the micro-scale the reflectance is characterized with the Cook-Torrance model and the distribution $D$ is assumed to be Gaussian, where the standard deviation represents the roughness; similarly at the mesoscale level roughness is approximated in terms of the standard deviation. The effect of the roughness at the microscale is assumed to be a blurring of perfect mirror reflections, whereas at the mesoscale it determines a permutation of the pixels. The surface is illuminated with a half-black, half-white image with a vertical edge, and the overall roughness is estimated by fitting a Gaussian filter that blurs the step-edge image to produce the observed one. To separate the roughness for the two different scales, all pixels are sorted by intensity and reshaped back in column-major order, thus removing the permutation induced by the mesoscale roughness; the slope of the segment obtained by averaging over the rows of the sorted image is used to estimate the microscale roughness. This approach can produce visually plausible results for highly glossy man-made indoor surfaces, including some paints, metals and plastics.

Riviere et al. [RPG15] propose a mobile reflectometry solution based on a mobile device's LCD panel as extended illumination source, statically mounted at a distance of $45 \mathrm{~cm}$ above a isotropic planar material sample, at normal incidence, in a dimly lit room (Figure 20(b)). The linear polarisation of the LCD panel is exploited for diffuse/specular separation, by taking two pictures of the sample with a differently orientated plastic sheet linear polariser in front of the device camera. Albedo, surface normals and specular roughness are estimated by illuminating the sample with the same lighting patterns described in $\left[\mathrm{GCP}^{*} 09\right]$. Due to the limited size of

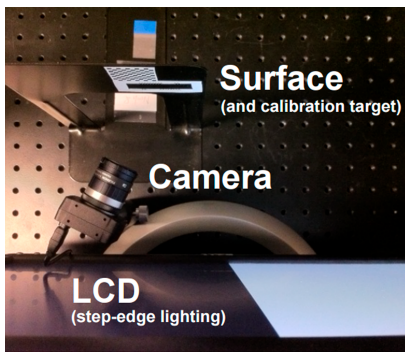

(a) Wang et al. [WSM11]

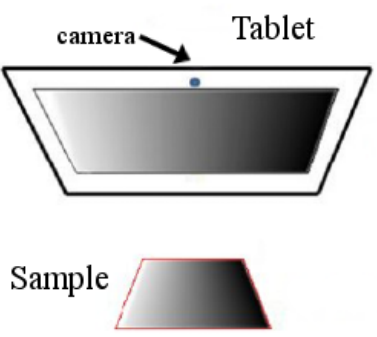

(b) Riviere et al. [RPG15]
Figure 20: Acquisition setup by Wang et al. [WSM11], based on step-edge illumination (c)2013 Association for Computing Machinery, Inc. Reprinted by permission (a). Mobile LCD measurement set-up for highly specular samples [RPG15] Copyright (C)2015 by John Wiley Sons, Inc. Reprinted by permission of John Wiley \& Sons, Inc. (b).

the LCD panel and the position of the front camera, this setup can only acquire $5 \mathrm{~cm} \times 5 \mathrm{~cm}$ area of the sample; for larger samples an appearance transfer approach, that relies on additional measurements under natural illumination, is used.

\subsection{Flash Illumination and other Capture Setups}

Backscattering data can be used to extract an appropriate distribution for microfacets BRDF models [AP07]. Based on this observation mobile devices equipped with a flash light, typically near the back camera, represent near-coaxial setups particularly useful to capture the backscatter surface reflectance to be fitted in a microfacets BRDF model [RPG15].

Riviere et al. [RPG15] mobile flash-based acquisition setup estimates the diffuse and specular albedo, surface normal and specular roughness of a planar material sample, with spatially varying isotropic surface reflectance. The back camera and flash light of a mobile device are used for a hand-held acquisition of a video in a dimly lit room, capturing data of the sample from several directions over the upper hemisphere (Figure 21). For reflectance calibration the diffuse grey squares of an X-Rite ColorChecker are used. The top view of the sample at normal incidence is used as a reference to register the other frames. To estimate the lighting and view directions the magnetometer/accelerometer sensors or 3D tracking can be used. The surface normal of each point is computed as the weighted average of the brightest reflection direction, the diffuse albedo is estimated as the trimmed median of the measured intensities, whereas the specular albedo is estimated from the hemispherical integral of the diffuse subtracted measurements. The specular roughness is obtained by fitting the observed backscattering profile to the [WMLT07] model (see Section 3.2). Some blurring in the reflectance maps can be introduced by misalignments and motion blur. The limited number of lighting directions suggests the use only for rough specular materials.

Aittala et al. [AWL15] mobile measurement setup for stationary materials consists of a single mobile device with on-board flash light (see Figure 22). Given a flash-no-flash image pair of a textured material of known characteristic size, a multi-stage reconstruction 


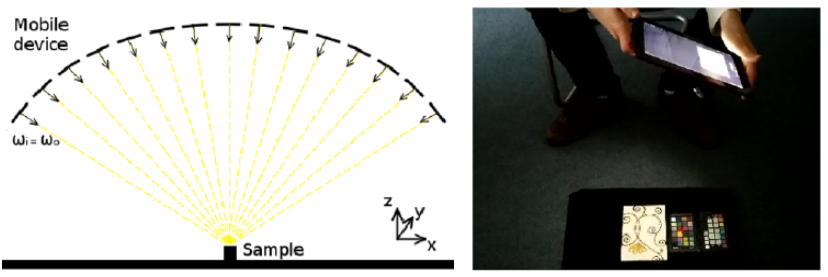

Figure 21: Riviere et al. [RPG15] mobile flash-based reflectance acquisition setup. Backscattering measurements with flash illumination (left); freeform acquisition using a tablet (right). Copyright (C)2015 by John Wiley Sons, Inc. Reprinted by permission of John Wiley \& Sons, Inc.

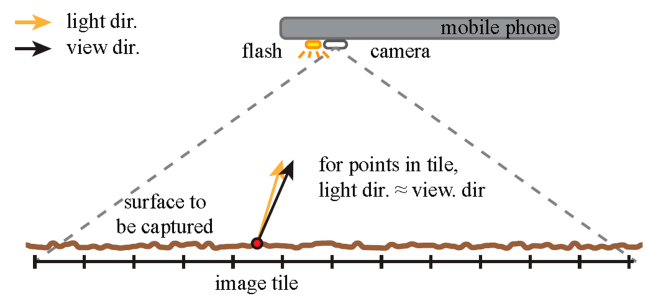

Figure 22: Geometry of the imaging setup proposed by Aittala et al. [AWL15] (c)2015 Association for Computing Machinery, Inc. Reprinted by permission.

pipeline allows to capture the full anisotropic SVBRDF. The input images are registered through a homography, computed from manually specified points of correspondence. The flash image provides an approximate retro-reflective measurement for each pixel, that combines the effect of surface normal and BRDF, whereas the other image is used as a guide to identify points on the surface with similar local reflectance. Since there is only one observation per pixel, it is assumed that multiple points on the surface share the same reflectance properies and that can be identified under ambient lighting to be combined together. The input is organised into regular tiles approximately of the same size of the repeating texture pattern, assumed to contain a random rearrangement of the same BRDF values. A master-tile is selected for relighting and lumitexels, (i.e. data structures to store the geometric and photometric data of one point [LKG ${ }^{*}$ 03]), are obtained for it. The lumitextels are regularised using a preliminary SVBRDF fit and augmented by transferring high-frequency detail from similarly lit tiles to reduce blurring. The augmented lumitexels are used in a non-linear optimizer to fit an analytic SVBRDF model and the solution is finally reverse-propagated to the full image. This setup limits the input to the retro-reflective slice of the BRDF, hence the Fresnel effect, shadowing and masking are assumed to have typical behaviour and modelled with the BRDF model A [BLPW14] (see Section 3.1). The camera field of view represents an upper limit on the width of the specular lobes which can be observed.

The idea that the variation of the reflectance over a target forms a low-dimensional manifold is exploited by Dong et al. [DWT*10], and describes a two-pass method to accelerate complex reflectance

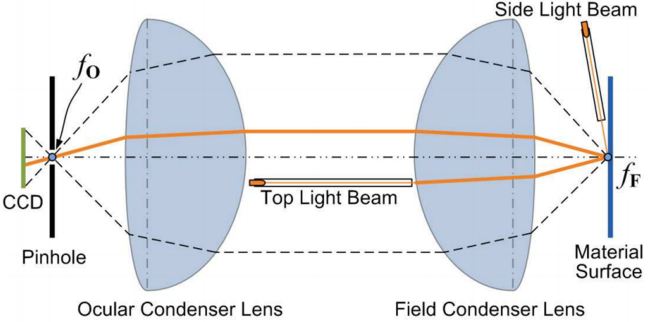

Figure 23: Optical design of the single point BRDF measurement device by Dong et al. [DWT 10] (C)2010 Association for Computing Machinery, Inc. Reprinted by permission.

capture, useful for both isotropic and anisotropic flat samples. During the first phase a set of high-resolution representative singlepoint BRDFs is captured using a hand-held device which scans over the sample. The device, schematised in Figure 23, consists of a pair of condenser lenses, a pinhole and a camera, aligned along the same optical axis by means of a lens tube. Six high brightness LED are used as light sources, with one light at the top and the remaining at the sides. The pinhole is placed at the focal plane of the ocular condenser lens such that the camera can image the light field of a single point on the surface, while the sample is placed at the focal plane of the field condenser lens. For each light a pair of $320 \times 240$ pixel pictures is taken, with different exposures in order to obtain a $240 \times 240$ HDR image, used for local reconstruction of BRDFs by convex linear combination in a small neighbourhood. The second phase captures a set of reflectance measurements densely over the surface, by means of a fixed DSLR camera and a hand-held light source, about 1.5 meters away from the sample and moved in a $2 \mathrm{D}$ plane opposite the sample itself; a mirrored ball is used to sample the incident lighting. Up to 200 pictures are acquired and used to map the manifold derived from the fist phase over the sample surface.

The measurement device presented in [HP03] is based on the principle of the kaleidoscope and consists of a tapered tube whose inner walls are lined with front-surface mirrors. A single camera captures the kaleidoscopic image, in which the subimages represent the same sample seen simultaneously from many different viewpoints. The sample is illuminated by a DLP projector, which shares the optical path with the camera by means of a $45^{\circ}$ beam splitter. The properties of the sample are measured through a sequence of pictures with different illumination images, which illuminate the sample from a known range of incoming directions due to the unique sequence of reflections from the kaleidoscopic walls. The advantages of this setting, suitable for BTFs and BSSRDFs, are the absence of moving parts which enables quick measurements and guarantee perfect registration of the measurements and the low cost; radiometric and geometric calibration need to be performed only once.

\section{Existing software for BRDF Data Fitting and Visualisation}

Currently the libraries of measured materials for rendering applications and the BRDF models included in rendering systems, 
generally defined as shaders [Sta99], do not fulfill modern material representation requirements and are computationally expensive. Moreover there is no universal BRDF model that can represent a wide range of materials, since most of them are designed to represent a specific set of phenomena. There are BRDF databases developed for specialised purposes, however to develop a new material a designer usually still needs to start from scratch to display it properly. Other than the previously mentioned MERL database [Mat03], the UTIA database is another source of publicly available BTFs [HM12] and BRDFs [FV14]; as for architectural materials there is an open source database [AB14] which provides measured data. Measured BRDF data usually cannot be used directly for rendering due to the noise in the measurement and needs to be interpolated or fitted to some analytic model.

Open source graphical user interfaces for BRDF shapes allow display and adjustment of various BRDF models. Examples of open source interfaces include BRDFLab [FPBP09], ALTA [BCP*15] and BRDF Explorer [MHH* 12] proposed by Disney. These applications handle analytical, measured and simulated reflectance models.

BRDF explorer [ $\left.\mathrm{MHH}^{*} 12\right]$ compares measured material models with existing analytic models and allows interactive adjustment of a few parameters. BRDFLab allows fitting measured data to analytical models and also combining different reflectance models, for example the Lafortune model and Blinn lobes. The software can also perform real-time optimisation of the models.

The BRDF analysis library ALTA $\left[\mathrm{BCP}^{*} 15\right]$ provides a set of instructions to perform fitting of the measured data to an analytical form, statistical analysis of data and offers a wide range of formats to export BRDF models. Additionally it provides functions to handle BRDF models, data to work with BRDF measurements and filters for fitting algorithms. Dupuy et al. [DHI* 15$]$ recently released a $C++$ library to fit a microfacet BRDF to an input material.

BRDF-Shop [FPBP09] is an interface developed to intuitively design arbitrary but physically plausible BRDFs, based on the extended Ward BRDF model. The interface provides control over the surface roughness and sets of brushes to add reflections and highlights. Finally, it is possible to adjust parameters and render the model under simulated lighting; a plug-in for Maya is available to the community.

\section{Conclusion}

We described some BRDF models, their representations and acquisition setups. For current state of the art representation and acquisition of BRDFs a high number of materials needs to be captured and represented for the Computer Graphics community. Each model is limited to a particular set of parameters which result in ability of the model to represent a specific material group. Even generalised models can not cover a broad range of materials nor variations of a material within one group. Therefore materials are mainly not inherited from the base material. Also an existing model assets can rarely be reused. At the moment material modelling involves a great deal of manual effort, ranging from completely manual creation of a material to fully automated acquired material which often cannot be used directly in rendering. The broad range of material models and complexity of the parameters requires from an artist an understanding of the underlying representation and material's micro/ macrostructure. There is no straight forward pipeline to measure and represent BRDF materials and not all of them can be captured with existing reflectance measurement setups. Some setups require samples of a specific size and it is not always possible to cut them precisely, Since acquisition devices generally work with planar samples, simple spherical and cylindrical samples. Moreover some layered materials like metallic car paint or varnished wood need to be captured and displayed properly to obtain realistic renderings. Their structure which contributes to obtaining complex reflectance properties, consists of an isotropic glossy lacquer covering anisotropic metal flakes and hence requires devices capable of dealing with anisotropy. Since BRDFs are the most recent development in the material representation field, the development of the appropriate resources continues. A physically accurate, consistent and intuitive material representation to represent materials efficiently, a comprehensive BRDF model would be beneficial for Computer Graphics. Although attempts to generalise reflectance models have been made by researchers, there is still no up to date universal material representation model that can fulfill such criteria and make it possible to standardise material representation.

\section{Biographies}

Dar' ya Guarnera is a $\mathrm{PhD}$ student at Loughborough University. She obtained her Postgraduate Certificate in Education from the Liverpool Hope University and first-class degree in Computer Science from the Liverpool Hope University. In 2006 she received Student of the year award at graduation from Liverpool Community College in 3D modelling. She also obtained BSc in Architecture from the Odessa State Academy of Civil Engineering and Architecture. Her interests includes 3D Architectural Visualisation, virtual materials, digital art and sculpture. She is a guest lecturer at Liverpool Hope University and part time lecturer at Liverpool Community College.

Giuseppe Claudio Guarnera is a Research Associate at Loughborough University. His research interests include Computer Vision, Pattern Recognition, Appearance Modelling and Computational Photography. He received his $\mathrm{PhD}$ in Computer Science from the University of Catania, with a doctoral dissertation in the area of Computer Vision and Pattern Recognition. Previously he was a postdoc at the University of Milano-Bicocca, working on high quality characterization of imaging devices and a Visiting Researcher at the University of Southern California Institute for Creative Technologies, working on surface normal estimation under controlled and uncontrolled lighting conditions. From 2006 to 2012 he was affiliated to the Image Processing Laboratory of the University of Catania, working on Shape Recognition, Medical Imaging and Image Processing.

Abhijeet Ghosh is a Lecturer in the Department of Computing at Imperial College London where he leads the Realistic Graphics and Imaging group. His main research interests are in appearance modelling, realistic rendering, computational photography and vision for graphics. Previously, he was a research assistant professor at the 
University of Southern California Institute for Creative Technologies where he worked on Light Stage based facial and appearance capture. Abhijeet received his $\mathrm{PhD}$ in computer science in 2007 from the University of British Columbia. His doctoral dissertation, "Realistic Materials and Illumination Environments", received an Alain Fournier Award. He currently holds a Royal Society Wolfson Research Merit Award and an EPSRC Early Career Fellowship at Imperial College London.

Cornelia Denk is currently working at BMW Group Research and Technology in Munich, Germany. A key part of her role is to establish a number of high profile international joint industryuniversity research initiatives for BMW Group. This work builds upon her prior experience gained while working at RTT in Munich as a Research Manager from 2008 to 2010 and as a Technology \& Innovation Manager from 2010 to 2011. In these roles she was instrumental in determining technical trends and innovation strategy, and responsible for establishing strategic partnerships both with industry and universities. Prior to this, she worked at RTT as a researcher on pre-computed global illumination algorithms and also gained international R\&D / Consulting experience in USA, Japan, South Korea, Singapore and China. Her specialist research areas are in Computer Graphics, Global Illumination, Augmented \& Virtual Reality, Process Validation, Sensors and I/O Devices. In addition to her work at BMW Group, she is involved in the Computer Graphics community through a range of activities, including service on the board of the Munich ACM SIGGRAPH Chapter, ACM-W Europe sub-committee and mentoring initiatives to help female students in technology.

Mashhuda Glencross is a lecturer at Loughborough University. She obtained her $\mathrm{PhD}$ in Computer Science from the University of Manchester. Prior to joining Loughborough, she worked at ARM in Cambridge and as a postdoc at the University of Manchester. Her research interests include virtual reality, 3D reconstruction/relighting, material appearance acquisition, novel user interfaces and human visual perception. At Loughborough, she leads the Future Digital Materials Lab. Her research is supported through three industrially funded research projects running in parallel. She is treasurer of the ACM Europe Council, chair of the ACM SIGGRAPH Professional and Student chapters committee and served as courses chair for SIGGRAPH 2014. She is currently also a member of the ACM Publication Board Conference Committee.

\section{References}

[X-16] X-RiTE, INC.: Ma98 portable spectrophotometer. http: //www.xrite.com/ma98-portable-spectrophotometer, 2016. Accessed: 2016-02-02.

[AB14] APIAN-BENNEWITZ P.: Building material examples (BME) BRDF and BSDF database. http://www.pab.eu/ gonio-photometer/demodata/bme/, 2014. Accessed: 2016$02-02$.

[AMHH08] AKenine-Möller T., Haines E., HofFman N.: RealTime Rendering 3rd Edition. A. K. Peters, Ltd., Natick, MA, USA, 2008.

[AP07] AshikHMin M., Premoze S.: Distribution-based BRDFs. Tech. rep., 2007.

[APSO0] ASHIKMIN M., PremoŽE S., SHIRlEy P.: A microfacet-based brdf generator. In Proceedings of the 27th Annual Conference on Computer Graphics and Interactive Techniques (New York, NY, USA, 2000),
SIGGRAPH '00, ACM Press/Addison-Wesley Publishing Co., pp. 6574.

[Arv95] ARVO J.: Applications of irradiance tensors to the simulation of non-lambertian phenomena. In Proceedings of the 22Nd Annual Conference on Computer Graphics and Interactive Techniques (New York, NY, USA, 1995), SIGGRAPH '95, ACM, pp. 335-342.

[AS00] ASHIKHMIN M., SHIRLEy P.: An anisotropic phong brdf model J. Graph. Tools 5, 2 (Feb. 2000), 25-32.

[ASMS01] Ashikhmin M., Shirley P., Marschner S., Stam J.: State of the art in modeling and measuring of surface reflection. In $A C M$ SIGGRAPH 2001 Courses (2001), ACM, p. 1.

[AWL13] Aittala M., Weyrich T., Lehtinen J.: Practical svbrdf capture in the frequency domain. ACM Trans. Graph. 32, 4 (July 2013), 110:1-110:12. URL: http://doi.acm.org/10.1145/ 2461912.2461978 .

[AWL15] AitTala M., Weyrich T., Lehtinen J.: Two-shot svbrdf capture for stationary materials. ACM Trans. Graph. 34, 4 (July 2015), 110:1-110:13. URL: http://doi.acm.org/10.1145/ 2766967.

[AZK08] Alldrin N., Zickler T., Kriegman D.: Photometric stereo with non-parametric and spatially-varying reflectance. In Computer Vision and Pattern Recognition, 2008. CVPR 2008. IEEE Conference on (2008), IEEE, pp. 1-8.

[BBP15] BARLA P., Belcour L., PACANOWSKi R.: In praise of an alternative brdf parametrization. In Workshop on Material Appearance Modeling (Darmstadt, Germany, June 2015), Proceedings of the Workshop on Material Appearance Modeling 2015.

[BCP*15] Belcour L., Courtes L., PaCANowski R., ET AL. ALTA: A BRDF Analysis Library. http: / / a lta.gforge.inria. fr/, 2013-2015.

[BEWW*08] Ben-Ezra M., Wang J., Wilburn B., Li X., Ma L.: An led-only brdf measurement device. In Computer Vision and Pattern Recognition, 2008. CVPR 2008. IEEE Conference on (June 2008), pp. 18. doi:10.1109/CVPR.2008.4587766.

[Bli77] BLINN J. F.: Models of light reflection for computer synthesized pictures. SIGGRAPH Comput. Graph. 11, 2 (July 1977), 192-198.

[BLPW14] BRADY A., LAWRENCE J., PEERS P., WEIMER W.: genbrdf: Discovering new analytic brdfs with genetic programming. ACM Trans. Graph. 33, 4 (July 2014), 114:1-114:11.

[BÖK11] BILGILI A., ÖZTÜRK A., KURT M.: A general brdf representation based on tensor decomposition. Computer Graphics Forum 30, 8 (2011), 2427-2439.

[Bra02] BRAND M.: Charting a manifold. In Advances in Neural Information Processing Systems 15 (2002), MIT Press, pp. 961-968.

[BSH12] Bagher M. M., Soler C., Holzschuch N.: Accurate fitting of measured reflectances using a shifted gamma micro-facet distribution. In Computer Graphics Forum (2012), vol. 31, Wiley Online Library, pp. 1509-1518.

[CBP04] Claustres L., Boucher Y., Paulin M.: Wavelet-based modeling of spectral bidirectional reflectance distribution function data. Optical Engineering 43, 10 (2004), 2327-2339.

[CDP*14] Chen G., Dong Y., Peers P., Zhang J., Tong X.: Reflectance scanning: Estimating shading frame and brdf with generalized linear light sources. ACM Trans. Graph. 33, 4 (July 2014), 117:1-117:11. URL: http://doi.acm.org/10.1145/ 2601097.2601180 .

[CGS06] Chen T., Goesele M., Seidel H.-P.: Mesostructure from specularity. In Computer Vision and Pattern Recognition, 2006 IEEE Computer Society Conference on (2006), vol. 2, IEEE, pp. 1825-1832.

[CJAMJ05] Clarberg P., JARosz W., AKenine-Möller T., JENSEN H. W.: Wavelet importance sampling: efficiently evaluating products of complex functions. In ACM Transactions on Graphics (TOG) (2005), vol. 24, ACM, pp. 1166-1175. 
[CMS87] CABRAL B., MAX N., SPRINGMEYER R.: Bidirectional reflection functions from surface bump maps. SIGGRAPH Comput. Graph. 21, 4 (Aug. 1987), 273-281.

[CP85] Clarke F., PARRY D.: Helmholtz reciprocity: its validity and application to reflectometry. Lighting Research and Technology 17, 1 (1985), 1-11.

[CPB03] Claustres L., PAulin M., Boucher Y.: Brdf measurement modelling using wavelets for efficient path tracing. Computer Graphics Forum (2003), 701-716.

[CT82] CoOK R. L., TorRAnCE K. E.: A reflectance model for computer graphics. ACM Transactions on Graphics (TOG) 1, 1 (1982), 7-24.

[CT91] ChurCh E. L., TAKACS P. Z.: Optimal estimation of finish parameters. In Proc. SPIE (1991), vol. 1530, pp. 71-85.

[CTL90] Church E. L., TAKacs P. Z., LeOnard T. A.: The prediction of brdfs from surface profile measurements. In Proc. SPIE (1990), vol. 1165, pp. 136-150.

[DHI*15] Dupuy J., Heitz E., IEhl J.-C., Poulin P., OstroMOUKHOV V.: Extracting microfacet-based brdf parameters from arbitrary materials with power iterations. Computer Graphics Forum 34, 4 (2015), 21-30.

[DI11] D'Eon E., IRVING G.: A quantized-diffusion model for rendering translucent materials. ACM Trans. Graph. 30, 4 (July 2011), 56:156:14.

[DLG13] Dong Y., Lin S., Guo B.: Material appearance modeling: A data-coherent approach. Springer, 2013.

[DR05] DORSEY J., RUSHMEIER H.: Digital modeling of the appearance of materials. In ACM SIGGRAPH 2005 Courses (2005), ACM, p. 1.

[DRS07] Dorsey J., RushmeIER H., Sillion F.: Digital Modeling of Material Appearance. Morgan Kaufmann, 2007.

[DS03] DACHSBACHER C., STAMMINGER M.: Translucent shadow maps. In Proceedings of the 14th Eurographics Workshop on Rendering (Aire-la-Ville, Switzerland, Switzerland, 2003), EGRW '03, Eurographics Association, pp. 197-201.

[Dür06] DÜR A.: An improved normalization for the ward reflectance model. Journal of Graphics, GPU, and Game Tools 11, 1 (2006), 51-59.

[DVGNK99] Dana K. J., VAN GinneKen B., NAYAR S. K., KoenDERINK J. J.: Reflectance and texture of real-world surfaces. ACM Transactions on Graphics (TOG) 18, 1 (1999), 1-34.

[DWd*08] DONNER C., WEYRICH T., D'EON E., RAMAMOORTHI R., RUSINKIEWICZ S.: A layered, heterogeneous reflectance model for acquiring and rendering human skin. ACM Trans. Graph. 27, 5 (Dec. 2008), 140:1-140:12.

[DWMG15] Dong Z., WAlter B., Marschner S., GreenberG D. P.: Predicting appearance from measured microgeometry of metal surfaces. ACM Trans. Graph. 35, 1 (Dec. 2015), 9:1-9:13.

[DWT*10] DONG Y., WANG J., TONG X., SNYDER J., LAN Y., BENEzRA M., Guo B.: Manifold bootstrapping for svbrdf capture. ACM Trans. Graph. 29, 4 (July 2010), 98:1-98:10. URL: http://doi. $\mathrm{acm}$. org/10.1145/1778765.1778835.

[EBJ*06] Edwards D., Boulos S., JOHnson J., Shirley P., ASHIKHMIN M., STARK M., WYMAN C.: The halfway vector disk for brdf modeling. ACM Trans. Graph. 25, 1 (Jan. 2006), 1-18.

[EDKM04] ERshov S., Durikovic R., Kolchin K., MYszKowsKi $\mathrm{K} .:$ Reverse engineering approach to appearance-based design of metallic and pearlescent paints. The Visual Computer 20, 8-9 (2004), 586-600.

[EKM01] ERshov S., Kolchin K., MyszKowski K.: Rendering pearlescent appearance based on paint-composition modelling. In Computer Graphics Forum (2001), vol. 20, Wiley Online Library, pp. 227238

[Fai05] FAIRChILD M. J.: Color Appearance Models. John Wiley \& Sons, 2005
[FCM*08] Francken Y., Cuypers T., Mertens T., Gielis J., BEKAERT P.: High quality mesostructure acquisition using specularities. In CVPR (2008).

[FH09] FILIP J., HAINDL M.: Bidirectional texture function modeling: A state of the art survey. Pattern Analysis and Machine Intelligence, IEEE Transactions on 31, 11 (2009), 1921-1940.

[FHV15] FILIP J., HAVLÍČEK M., VÁVRA R.: Adaptive highlights stencils for modeling of multi-axial brdf anisotropy. The Visual Computer (2015), 1-11.

[Foo97] Foo S. C.: A gonioreflectometer for measuring the bidirectional reflectance of material for use in illumination computation. $\mathrm{PhD}$ thesis Cornell University, 1997.

[Fou95] FOURNIER A.: Separating reflection functions for linear radiosity. In Rendering Techniques 95, Eurographics. Springer Vienna, 1995, pp. 296-305.

[FPBP09] Forés A., Pattanaik S. N., Bosch C., Pueyo X.: Brdflab: A general system for designing brdfs. In XIX Congreso Espa nol de Informática Gráfica (CEIG09), San Sebastián, Spain (2009).

[FV14] FILIP J., VÁVRA R.: Template-based sampling of anisotropic brdfs. Computer Graphics Forum (Proceedings of Pacific Graphics 2014) (2014)

[FVK14] FiliP J., VÁvra R., KRUPiČKa M.: Rapid material appearance acquisition using consumer hardware. Sensors 14, 10 (2014), 19785-19805.

[GAHO07] Ghosh A., Achutha S., Heidrich W., O'Toole M. Brdf acquisition with basis illumination. In Computer Vision, 2007. ICCV 2007. IEEE 11th International Conference on (Oct 2007), pp. 1-8. doi:10.1109/ICCV.2007.4408935.

[GBS14] Guarnera G. C., Bianco S., Schettini R.: Absolute colorimetric characterization of a dslr camera. In Proc. SPIE (2014), vol. 9023, pp. 90230U-90230U-7.

[GCHS05] Goldman D. B., Curless B., Hertzmann A., Seitz S. M.: Shape and spatially-varying brdfs from photometric stereo. In Proceedings of the Tenth IEEE International Conference on Computer Vision (ICCV'05) Volume 1 - Volume 01 (Washington, DC, USA, 2005), ICCV '05, IEEE Computer Society, pp. 341-348.

[GCP*09] Ghosh A., Chen T., Peers P., Wilson C. A., Debevec P.: Estimating specular roughness and anisotropy from second order spherical gradient illumination. In Proceedings of the Twentieth Eurographics Conference on Rendering (Aire-la-Ville, Switzerland, Switzerland, 2009), EGSR'09, Eurographics Association, pp. 1161-1170.

[GCP*10] Ghosh A., Chen T., Peers P., Wilson C. A., Debevec P.: Circularly polarized spherical illumination reflectometry. ACM Trans. Graph. 29, 6 (Dec. 2010), 162:1-162:12.

[Geo03] GEORGHIADES A. S.: Recovering 3-d shape and reflectance from a small number of photographs. In Proceedings of the 14th Eurographics Workshop on Rendering (Aire-la-Ville, Switzerland, Switzerland, 2003), EGRW '03, Eurographics Association, pp. 230-240.

[GHAO10] Ghosh A., Heidrich W., Achutha S., O'Toole M.: A basis illumination approach to brdf measurement. International journal of computer vision 90, 2 (2010), 183-197.

[GHP*08] Ghosh A., Hawkins T., Peers P., Frederiksen S., DeBEVEC P.: Practical modeling and acquisition of layered facial reflectance. In ACM Transactions on Graphics (TOG) (2008), vol. 27 ACM, p. 139

[GLL*04] Goesele M., Lensch H. P. A., LAng J., Fuchs C., SeIDEL H.-P.: Disco: Acquisition of translucent objects. ACM Trans. Graph. 23, 3 (Aug. 2004), 835-844.

[GLS04] Goesele M., LENSCH H., SEIDEL H.-P.: Validation of color managed $3 \mathrm{~d}$ appearance acquisition. In Color and Imaging Conference (2004), vol. 2004, Society for Imaging Science and Technology, pp. 265270. 
[GMD10] GEISLER-Moroder D., DÃIJR A.: A new ward brdf model with bounded albedo. Computer Graphics Forum 29, 4 (2010), 13911398 .

[GPDG12] Guarnera G., Peers P., Debevec P., Ghosh A.: Estimating surface normals from spherical stokes reflectance fields. In Computer Vision - ECCV 2012. Workshops and Demonstrations, Fusiello A., Murino V., Cucchiara R., (Eds.), vol. 7584 of Lecture Notes in Computer Science. Springer Berlin Heidelberg, 2012, pp. 340-349.

[GTGB84] Goral C. M., Torrance K. E., Greenberg D. P., BatTAILE B.: Modeling the interaction of light between diffuse surfaces. SIGGRAPH Comput. Graph. 18, 3 (Jan. 1984), 213-222.

[GTHD03] Gardner A., Tchou C., Hawkins T., Debevec P.: Linear light source reflectometry. ACM Trans. Graph. 22, 3 (July 2003), 749-758. URL: http://doi.acm.org/10.1145/882262. 882342 .

[Hai91] HaINES E. A.: Beams o' light: Confessions of a hacker. SIGGRAPH ăĂŹ91 Course Notes - Frontiers in Rendering (July 1991).

[HBV03] HAO X., BABY T., VARSHNEY A.: Interactive subsurface scattering for translucent meshes. In Proceedings of the 2003 Symposium on Interactive 3D Graphics (New York, NY, USA, 2003), I3D '03, ACM, pp. 75-82.

[HDCD15] Heitz E., Dupuy J., Crassin C., Dachsbacher C.: The sggx microflake distribution. ACM Trans. Graph. 34, 4 (July 2015), 48:1-48:11

[Hei14] HeITZ E.: Understanding the masking-shadowing function in microfacet-based brdfs. Journal of Computer Graphics Techniques (JCGT) 3, 2 (June 2014), 48-107.

[HF13] HaINDL M., FILIP J.: Spatially varying bidirectional reflectance distribution functions. In Visual Texture, Advances in Computer Vision and Pattern Recognition. Springer London, 2013, pp. 119-145.

[HK93] HANRAHAN P., KRUEGER W.: Reflection from layered surfaces due to subsurface scattering. In Proceedings of the 20th Annual Conference on Computer Graphics and Interactive Techniques (New York, NY, USA, 1993), SIGGRAPH '93, ACM, pp. 165-174.

[HLHZ08] Holroyd M., LAWrence J., Humphreys G., ZiCKLER T.: A photometric approach for estimating normals and tangents. ACM Transactions on Graphics (Proceedings of SIGGRAPH Asia 2008) 27, 5 (2008).

[HM12] HAINDL M. FILIP J. V. R.: Digital material appearance: the curse of tera-bytes. ERCIM News, 90 (2012), 49-50.

[HP03] HAN J. Y., PERLIN K.: Measuring bidirectional texture reflectance with a kaleidoscope. ACM Trans. Graph. 22, 3 (July 2003), 741-748.

[HR76] HSIA J. J., RICHMOND J. C.: Bidirectional reflectometry. part i: A high resolution laser bidirectional reflectometer with results on several optical coatings. Journal of Reseach of the National Bureau of Standards-A. Physics and Chemistry A 80 (1976), 189-205

[HS05] Hertzmann A., SeItz S. M.: Example-based photometric stereo: Shape reconstruction with general, varying brdfs. Pattern Analysis and Machine Intelligence, IEEE Transactions on 27, 8 (2005), 1254 1264.

[HTSG91] He X. D., Torrance K. E., Sillion F. X., Greenberg D. P.: A comprehensive physical model for light reflection. SIGGRAPH Comput. Graph. 25, 4 (July 1991), 175-186.

[JAM*10] JAKOB W., ARbreE A., Moon J. T., BAlA K., MARSCHNER S.: A radiative transfer framework for rendering materials with anisotropic structure. ACM Trans. Graph. 29, 4 (July 2010), 53:1-53:13

[JHY* 14] JaKob W., Hašan M., YAn L.-Q., LaWrence J., RAMAMOORTHI R., MARSCHNER S.: Discrete stochastic microfacet models. ACM Trans. Graph. 33, 4 (July 2014), 115:1-115:10. doi: $10.1145 / 2601097.2601186$
[JMLH01] JENSEN H. W., MARSChNer S. R., LEVOY M., HANRAHAN P.: A practical model for subsurface light transport. In Proceedings of the 28th Annual Conference on Computer Graphics and Interactive Techniques (New York, NY, USA, 2001), SIGGRAPH '01, ACM, pp. 511-518.

[KK89] KAJIYA J. T., KAY T. L.: Rendering fur with three dimensional textures. SIGGRAPH Comput. Graph. 23, 3 (July 1989), 271-280.

[KK08] KIM M. H., KAUTZ J.: Characterization for high dynamic range imaging. Computer Graphics Forum 27, 2 (2008), 691-697.

[KM99] KAUTZ J., McCool M. D.: Interactive rendering with arbitrary brdfs using separable approximations. In Rendering Techniques 99. Springer, 1999 , pp. 247-260.

[Kon16] Konica Minolta Sensing Americas, InC: Dms 201-505-803. http://sensing.konicaminolta.us/ technologies/display-measurement/, 2016. Accessed: 2016-02-02.

[KRP*15] Klehm O., Rousselle F., Papas M., BRadley D., Hery C., Bickel B., JARosz W., BeEler T.: Recent advances in facial appearance capture. Computer Graphics Forum (Proceedings of Eurographics) 34, 2 (May 2015), 709-733.

[KSKK10] KURT M., SZIRMAY-KALOS L., KŘIVÁNEK J.: An anisotropic brdf model for fitting and monte carlo rendering. SIGGRAPH Comput. Graph. 44, 1 (Feb. 2010), 3:1-3:15.

[KSZ* 15] Khungurn P., Schroeder D., ZhaO S., Bala K. MARSCHNER S.: Matching real fabrics with micro-appearance models. ACM Trans. Graph. 35, 1 (Dec. 2015), 1:1-1:26

[LBAD*06] Lawrence J., Ben-Artzi A., DeCoro C., Matusik W., Pfister H., Ramamoorthi R., Rusinkiewicz S.: Inverse shade trees for non-parametric material representation and editing. In ACM SIGGRAPH 2006 Papers (New York, NY, USA, 2006), SIGGRAPH '06, ACM, pp. 735-745.

[Lew94] LEWIS R. R.: Making shaders more physically plausible. Com puter Graphics Forum 13, 2 (1994), 109-120.

[LF97] LALONDE P., FouRnier A.: A wavelet representation of reflectance functions. IEEE Transactions on Visualization and Computer Graphics 3, 4 (Oct. 1997), 329-336.

[LFTG97] Lafortune E. P. F., Foo S.-C., TORRance K. E., GREENBERG D. P.: Non-linear approximation of reflectance functions In Proceedings of the 24th Annual Conference on Computer Graphics and Interactive Techniques (New York, NY, USA, 1997), SIGGRAPH '97, ACM Press/Addison-Wesley Publishing Co., pp. 117-126.

[LFTW06] Li H., Foo S.-C., TORRAnCE K. E., Westin S. H.: Automated three-axis gonioreflectometer for computer graphics applications. Optical Engineering 45, 4 (2006), 043605-043605.

[Lig16] LIGHT TEC: Reflet. http://www.lighttec.fr/ scattering_instrument.html, 2016. Accessed: 2016-02-02.

[LKG*03] Lensch H. P. A., Kautz J., Goesele M., Heidrich W., SEIDEL H.-P.: Image-based reconstruction of spatial appearance and geometric detail. ACM Trans. Graph. 22, 2 (Apr. 2003), 234-257.

[LKYU12] LöW J., Kronander J., Ynnerman A., Unger J.: Brdf models for accurate and efficient rendering of glossy surfaces. ACM Transactions on Graphics (TOG) 31, 1 (2012), 9.

[LRR04] LAWRENCE J., RUSINKIEWICZ S., RAMAMOORTHI R.: Efficient brdf importance sampling using a factored representation. In $A C M$ Transactions on Graphics (TOG) (2004), vol. 23, ACM, pp. 496-505.

[LW94] Lafortune E. P., Willems Y. D.: Using the Modified Phong Reflectance Model for Physically Based Rendering. Tech. rep., 1994.

[MAA01] McCool M. D., ANG J., AHMAD A.: Homomorphic factorization of brdfs for high-performance rendering. In Proceedings of the 28th Annual Conference on Computer Graphics and Interactive Techniques (New York, NY, USA, 2001), SIGGRAPH '01, ACM, pp. 171178 
[Mat03] Matusik W.: A data-driven reflectance model. $\mathrm{PhD}$ thesis, Massachusetts Institute of Technology, 2003.

[MD98] MARSCHNER S. R., D P.: Inverse Rendering for Computer Graphics. Tech. rep., 1998.

[MGW01] Malzbender T., Gelb D., Wolters H.: Polynomial texture maps. In Proceedings of the 28th Annual Conference on Computer Graphics and Interactive Techniques (New York, NY, USA, 2001), SIGGRAPH '01, ACM, pp. 519-528.

[MHH* 12] McAuley S., Hill S., Hoffman N., Gotanda Y., Smits B., Burley B., Martinez A.: Practical physically-based shading in film and game production. In ACM SIGGRAPH 2012 Courses (New York, NY, USA, 2012), SIGGRAPH '12, ACM, pp. 10:1-10:7.

[MHP*07] Ma W.-C., Hawkins T., Peers P., Chabert C.-F., Weiss M., DEBEVEC P.: Rapid acquisition of specular and diffuse normal maps from polarized spherical gradient illumination. In Proceedings of the 18th Eurographics Conference on Rendering Techniques (Aire-la-Ville, Switzerland, Switzerland, 2007), EGSR'07, Eurographics Association, pp. 183-194.

[MMS*04] Müller G., Meseth J., Sattler M., Sarlette R., KLEIN R.: Acquisition, synthesis and rendering of bidirectional texture functions. In Eurographics 2004, State of the Art Reports (Sept. 2004), Schlick C., Purgathofer W., (Eds.), INRIA and Eurographics Association, pp. 69-94.

[MPBM03a] Matusik W., Pfister H., Brand M., McMillan L.: A data-driven reflectance model. ACM Trans. Graph. 22, 3 (July 2003), 759-769.

[MPBM03b] Matusik W., Pfister H., Brand M., McMillan L. Efficient isotropic brdf measurement. In Proceedings of the 14th Euro graphics Workshop on Rendering (Aire-la-Ville, Switzerland, Switzerland, 2003), EGRW '03, Eurographics Association, pp. 241-247.

[MSY07] Mukaigawa Y., Sumino K., Yagi Y.: Multiplexed illumination for measuring brdf using an ellipsoidal mirror and a projector. In Computer Vision-ACCV 2007. Springer, 2007, pp. 246-257.

[MU12] Montes S. R., UREÑA A. C.: An overview of brdf models.

[MWL*99] Marschner S. R., Westin S. H., Lafortune E. P., Torrance K. E., GreEnberG D. P.: Image-based brdf measurement including human skin. In Rendering Techniques 99. Springer, 1999 pp. 131-144.

[NDM05] NGan A., Durand F., Matusik W.: Experimental analysis of brdf models. In Proceedings of the Sixteenth Eurographics Conference on Rendering Techniques (Aire-la-Ville, Switzerland, Switzerland, 2005), EGSR'05, Eurographics Association, pp. 117-126. doi: $10.2312 / \mathrm{EGWR} / \mathrm{EGSR} 05 / 117-126$.

[NL11] Nishino K., LOMBARDI S.: Directional statistics-based reflectance model for isotropic bidirectional reflectance distribution functions. J. Opt. Soc. Am. A 28, 1 (Jan 2011), 8-18.

[NNSK99] Neumann L., Neumannn A., SzIRmay-Kalos L.: Compact metallic reflectance models. Computer Graphics Forum 18, 3 (1999), 161-172.

[NRH*77] Nicodemus F., Richmond J., Hsia J., GinsberG I., LIMPERIS T.: Geometrical considerations and nomenclature for reflectance, natl. Bur. Stand. Rep., NBS MN-160 (1977).

[NZV*11] NAIK N., ZhaO S., Velten A., RASKaR R., Bala K. Single view reflectance capture using multiplexed scattering and timeof-flight imaging. ACM Trans. Graph. 30, 6 (Dec. 2011), 171:1-171:10. URL: http://doi.acm.org/10.1145/2070781.2024205.

[OKBG08] Ozturk A., Kurt M., Bilgili A., Gungor C.: Linear approximation of bidirectional reflectance distribution functions. Computers \& Graphics 32, 2 (2008), 149-158.

[ON94] OREN M., NAYAR S. K.: Generalization of lambert's reflectance model. In Proceedings of the 21st annual conference on Computer graphics and interactive techniques (1994), ACM, pp. 239-246.
[Ops16] OPSIRA: gonio'2pi. http://www.opsira.de/en/ products/optical-measurement-systems/, 2016. Accessed: 2016-02-02.

[PFTV88] Press W. H., Flannery B. P., Teukolsky S. A., VetTERLING W. T.: Numerical Recipes in C: The Art of Scientific Computing. Cambridge University Press, New York, NY, USA, 1988.

[PH89] Perlin K., Hoffert E. M.: Hypertexture. SIGGRAPH Comput. Graph. 23, 3 (July 1989), 253-262.

[Pho75] Phong B. T.: Illumination for computer generated pictures. Commun. ACM 18, 6 (June 1975), 311-317.

[PSCS*12] PaCanowski R., Salazar Celis O., Schlick C., Granier X., Poulin P., CUYT A.: Rational brdf. Visualization and Computer Graphics, IEEE Transactions on 18, 11 (Nov 2012), 18241835 .

[Rad16] RADIANT Vision Systems: Imaging spheres. http: //www.radiantvisionsystems.com/products/, 2016. Accessed: 2016-02-02.

[RCH12] Riviere N., CeOlato R., Hespel L.: Multispectral polarized brdf: design of a highly resolved reflectometer and development of a data inversion method. Opt. Appl 42 (2012).

[RLCP] Rushmeier H., Lockerman Y. D., Cartwright L., PitERA D.: Experiments with a low-cost system for computer graphics material model acquisition.

[RMS*08] Rump M., Müller G., SARlette R., Koch D., KleiN R.: Photo-realistic rendering of metallic car paint from image-based measurements. Computer Graphics Forum 27, 2 (Apr. 2008), 527-536.

[RPG15] Riviere J., PeErs P., Ghosh A.: Mobile surface reflectometry. Computer Graphics Forum (2015), n/a-n/a. URL: http: //dx.doi.org/10.1111/cgf.12719.

[Rus98] RUSINKIEWICZ S.: A new change of variables for efficient brdf representation. In Rendering Techniques 98, Drettakis G., Max N., (Eds.), Eurographics Book Series. Springer-Verlag, Wien, Austria, 1998. Proceedings of the Workshop held in Vienna, Austria, between Jyne 29th and July 1st 1998.

[RVZ08] ROMEIRo F., VASILYEV Y., ZICKLER T.: Passive reflectometry. In Proceedings of the 10th European Conference on Computer Vision: Part IV (Berlin, Heidelberg, 2008), ECCV '08, Springer-Verlag, pp. $859-872$.

[RWS*11] Ren P., Wang J., SNyder J., Tong X., Guo B.: Pocket reflectometry. ACM Trans. Graph. 30, 4 (July 2011), 45:1-45:10.

[SAS05] Stark M., Arvo J., Smits B.: Barycentric parameterizations for isotropic brdfs. Visualization and Computer Graphics, IEEE Transactions on 11, 2 (March 2005), 126-138.

[Sch94] SCHLICK C.: An inexpensive brdf model for physically-based rendering. Computer Graphics Forum 13, 3 (1994), 233-246.

[Sch16] SChMitT IndustriEs, InC.: Casi scatterometer. http://sms.schmitt-ind.com/products casi-scatterometer/, 2016. Accessed: 2016-02-02.

[SDSG13] Schregle R., Denk C., Slusallek P., Glencross M.: Grand challenges: Material models in automotive. In Proceedings of the Eurographics 2013 Workshop on Material Appearance Modeling: Issues and Acquisition (Aire-la-Ville, Switzerland, Switzerland, 2013), MAM '13, Eurographics Association, pp. 1-6.

[Smi67] SмIтH B.: Geometrical shadowing of a random rough surface. Antennas and Propagation, IEEE Transactions on 15, 5 (1967), 668671.

[Sta99] Stamminger M.: Finite element methods for global illumination computations. Herbert Utz Verlag, 1999.

[Sur16] Surface Optics CORPORATION: Soc-200 bdr bidirectional reflectometer. https://surfaceoptics.com/products/ reflectometers-emissometers/soc200/, 2016. Accessed: 2016-02-02 
[SZC*07] Sun X., Zhou K., Chen Y., Lin S., Shi J., Guo B.: Interactive relighting with dynamic brdfs. ACM Trans. Graph. 26, 3 (July 2007).

[TFG*13] Tunwattanapong B., Fyffe G., Graham P., Busch J., Yu X., Ghosh A., Debevec P.: Acquiring reflectance and shape from continuous spherical harmonic illumination. ACM Trans. Graph 32, 4 (July 2013), 109:1-109:12. URL: http: / / doi . acm . org/10 . $1145 / 2461912.2461944$

[TR75] Trowbridge T. S., ReITZ K. P.: Average irregularity representation of a rough surface for ray reflection. J. Opt. Soc. Am. 65, 5 (May 1975), 531-536.

[TS67] TORRANCE K. E., SPARrow E. M.: Theory for off-specular reflection from roughened surfaces. JOSA 57, 9 (1967), 1105-1112.

[Tuc66] TUCKER L.: Some mathematical notes on three-mode factor analysis. Psychometrika 31, 3 (1966), 279-311.

[Vea97] Veach E.: Robust Monte Carlo Methods for Light Transport Simulation. PhD thesis, Stanford University, 1997.

[War92] WARD G. J.: Measuring and modeling anisotropic reflection. SIGGRAPH Comput. Graph. 26, 2 (July 1992), 265-272.

[WAT92] Westin S. H., Arvo J. R., Torrance K. E.: Predicting reflectance functions from complex surfaces. SIGGRAPH Comput. Graph. 26, 2 (July 1992), 255-264.

[WDR11] WU H., DORSEY J., RUSHMEIER H.: A sparse parametric mixture model for btf compression, editing and rendering. In Computer Graphics Forum (2011), vol. 30, Wiley Online Library, pp. 465-473.

[WEV02] WARd G., EydelberG-Vileshin E.: Picture perfect rgb rendering using spectral prefiltering and sharp color primaries. In Proceedings of the 13th Eurographics Workshop on Rendering (Aire-laVille, Switzerland, Switzerland, 2002), EGRW '02, Eurographics Association, pp. 117-124.

[WKB12] WARD G., KURT M., BONNEEL N.: Framework for Sharing and Rendering Real-World Bidirectional Scattering Distribution Functions. Tech. Rep. LBNL-5954E, Lawrence Berkeley National Laboratory, October 2012.

[WKB14] WARD G., KURT M., BONNEEL N.: Reducing anisotropic bsdf measurement to common practice. In Proceedings of the Eurographics 2014 Workshop on Material Appearance Modeling: Issues and Acquisition (Aire-la-Ville, Switzerland, Switzerland, 2014), MAM '14, Eurographics Association, pp. 5-8.

[WLL*09] Weyrich T., LAWRENCE J., LENSCH H., RUSINKIEWICZ S., ZICKLER T.: Principles of appearance acquisition and representa-

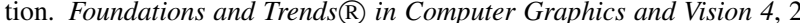
(2009), 75-191.

[WLT04] Westin S. H., Li H., TorRance K. E.: A comparison of four brdf models. In Eurographics Symposium on Rendering. pags (2004), pp. 1-10.

[WMLt07] Walter B., Marschner S. R., Li H., TORRance K. E.: Microfacet models for refraction through rough surfaces. In Proceedings of the 18th Eurographics conference on Rendering Techniques (2007), Eurographics Association, pp. 195-206.

[Woo80] Woodham R. J.: Photometric method for determining surface orientation from multiple images. Optical Engineering 19, 1 (1980), 191139-191139-

[WS82] Wyszecki G., Stiles W. S.: Color science: Concepts and Methods, Quantitative Data and Formulae, vol. 8. 1982.

[WSM11] Wang C.-P., Snavely N., Marschner S.: Estimating dual-scale properties of glossy surfaces from step-edge lighting. In Proceedings of the 2011 SIGGRAPH Asia Conference (New York, NY, USA 2011), SA '11, ACM, pp. 172:1-172:12. URL: http://doi.acm. org/10.1145/2024156.2024206

[WW07] WEIDLICH A., WILKIE A.: Arbitrarily layered micro-facet surfaces. In Proceedings of the 5th International Conference on Computer Graphics and Interactive Techniques in Australia and Southeast Asia (New York, NY, USA, 2007), GRAPHITE '07, ACM, pp. 171-178.
[XCL*01] XU Y.-Q., CHEN Y., LIN S., ZHONG H., WU E., GUO B., SHum H.-Y.: Photorealistic rendering of knitwear using the lumislice. In Proceedings of the 28th Annual Conference on Computer Graphics and Interactive Techniques (New York, NY, USA, 2001), SIGGRAPH '01, ACM, pp. 391-398.

[ZBB*15] Zubiaga C. J., Belcour L., Bosch C., Muñoz A., BARLA P.: Statistical analysis of bidirectional reflectance distribution functions. In Measuring, Modeling, and Reproducing Material Appearance 2015 (San Francisco, United States, Feb. 2015).

[ZJMB11] Zhao S., JaKob W., Marschner S., Bala K.: Building volumetric appearance models of fabric using micro ct imaging. $A C M$ Trans. Graph. 30, 4 (July 2011), 44:1-44:10.

[ZREB06] Zickler T., RAMAMOORThi R., ENRIQUE S., BelHUMEUR P. N.: Reflectance sharing: Predicting appearance from a sparse set of images of a known shape. IEEE Trans. Pattern Anal. Mach. Intell. 28, 8 (Aug. 2006), 1287-1302. 